Prepared for the U.S. Department of Energy

under Contract DE-AC05-76RL01830

\title{
Hanford Site Environmental Surveillance Master Sampling Schedule for Calendar Year 2011
}

\author{
LE Bisping
}

January 2011

Pacific Northwest

NATIONAL LABORATORY

Proudly Operated by Battelle Since 1965 


\title{
DISCLAIMER
}

This report was prepared as an account of work sponsored by an agency of the United States Government. Neither the United States Government nor any agency thereof, nor Battelle Memorial Institute, nor any of their employees, makes any warranty, express or implied, or assumes any legal liability or responsibility for the accuracy, completeness, or usefulness of any information, apparatus, product, or process disclosed, or represents that its use would not infringe privately owned rights. Reference herein to any specific commercial product, process, or service by trade name, trademark, manufacturer, or otherwise does not necessarily constitute or imply its endorsement, recommendation, or favoring by the United States Government or any agency thereof, or Battelle Memorial Institute. The views and opinions of authors expressed herein do not necessarily state or reflect those of the United States Government or any agency thereof.

\author{
PACIFIC NORTHWEST NATIONAL LABORATORY \\ operated by \\ BATTELLE \\ for the \\ UNITED STATES DEPARTMENT OF ENERGY \\ under Contract DE-AC05-76RL01830
}

Printed in the United States of America
Available to DOE and DOE contractors from the
Office of Scientific and Technical Information,
P.O. Box 62, Oak Ridge, TN 37831-0062;
ph: (865) 576-8401
fax: $(865) 576-5728$
email: reports@adonis.osti.gov

\begin{abstract}
Available to the public from the National Technical Information Service, U.S. Department of Commerce, 5285 Port Royal Rd., Springfield, VA 22161 ph: (800) 553-6847 fax: $(703) 605-6900$ email: orders@ntis.fedworld.gov online ordering: http://www.ntis.gov/ordering.htm
\end{abstract}

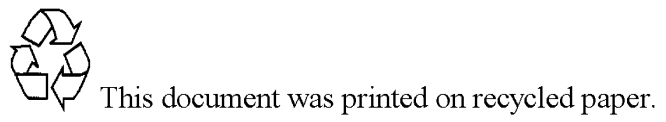




\section{Hanford Site Environmental Surveillance Master Sampling Schedule for Calendar Year 2011}

LE Bisping

January 2011

Prepared for

the U.S. Department of Energy

under Contract DE-AC05-76RL01830

Pacific Northwest National Laboratory

Richland, Washington 99352 


\section{Summary}

Environmental surveillance of the Hanford Site and surrounding areas is conducted by Pacific Northwest National Laboratory for the U.S. Department of Energy (DOE). ${ }^{1}$ Sampling is conducted to evaluate levels of radioactive and nonradioactive pollutants in the Hanford Site environs, as required in DOE Order 450.1A, "Environmental Protection Program," and DOE Order 5400.5, "Radiation Protection of the Public and the Environment.",3 The environmental surveillance sampling design is described in the Hanford Site Environmental Monitoring Plan, United States Department of Energy, Richland Operations Office (DOE/RL-91-50). ${ }^{4}$

This document contains the calendar year 2011 schedule for the routine collection of samples for the Surface Environmental Surveillance Project (SESP) and the Drinking Water Monitoring Project (DWMP). Each section includes sampling locations, sampling frequencies, sample types, and analyses to be performed. In some cases, samples are scheduled on a rotating basis. If a sample will not be collected in 2011, the anticipated year for collection is provided. Maps showing approximate sampling locations are included for media scheduled for collection in 2011.

\section{Surface Environmental Surveillance Project Sampling}

The SESP is a multimedia environmental surveillance effort to measure the concentrations of radionuclides and chemicals in environmental media to demonstrate compliance with applicable environmental quality standards and public exposure limits, and assess environmental impacts. Project personnel annually collect selected samples of ambient air, surface water, agricultural products, fish, wildlife, and sediments. Soil and vegetation samples are collected approximately every 5 years. Analytical capabilities include the measurement of radionuclides at environmental concentrations and in selected media; and nonradiological constituents including metals, anions, volatile organic compounds, and total organic carbon.

\section{Drinking Water Monitoring Project Sampling}

Mission Support Alliance, LLC is responsible for monitoring the quality of drinking water supplied by DOE to its onsite facilities in accordance with federal and state regulations. Pacific Northwest National Laboratory conducts radiological monitoring of onsite drinking water for Mission Support Alliance, LLC concurrent with SESP activities to promote sampling efficiency and consistency; use expertise developed over the years; and reduce costs associated with management, sample collection, procedure development, analytical contracting, data management, quality control, and reporting.

\footnotetext{
${ }^{1}$ Pacific Northwest National Laboratory is operated by Battelle for the U.S. Department of Energy.

${ }^{2}$ DOE Order 450.1A. 2008. "Environmental Protection Program." U.S. Department of Energy, Washington, D.C.

${ }^{3}$ DOE Order 5400.5, Chg 2. 1993. "Radiation Protection of the Public and the Environment." U.S. Department of Energy, Washington, D.C.

${ }^{4}$ DOE/RL-91-50, Rev. 4. 2008. Environmental Monitoring Plan, United States Department of Energy, Richland Operations Office. U.S. Department of Energy, Richland Operations Office, Richland, Washington.
} 


\section{Data Management}

The Hanford Environmental Information System (HEIS) database is used as a repository for data gathered during environmental surveillance activities at the Hanford Site. For ease in retrieving SESP or drinking water data from the HEIS database, the majority of the location names in this document are the location names used in the database. ${ }^{5}$

\section{Schedule Changes}

This schedule is subject to modification during the year in response to changes in Hanford Site operations, program requirements, and the nature of the observed results. Operational limitations such as weather, mechanical failures, sample availability, and other factors may also impact scheduled sampling. Therefore, this document may not be an accurate record of samples collected during the year.

\section{Multi-Agency Samples}

By joint agreement, some samples are collected by SESP personnel and provided to the Washington State Department of Health. All planned cooperative sampling efforts are indicated in this schedule.

\section{Additional Information}

Questions relating to the content of this document can be directed to either

T. M. (Ted) Poston, Manager

Surface Environmental Surveillance Project

(509) 372-6900

or

L. E. Bisping, Manager,

Drinking Water Monitoring Project

(509) 371-7121

${ }^{5}$ HEIS. 1989. Hanford Environmental Information System. Environmental Database Management, CH2M HILL Plateau Remediation Company, Richland, Washington. 


\section{Acronyms and Symbols}

\section{Acronyms}

$\begin{array}{ll}\text { ALE } & \text { Fitzner/Eberhardt Arid Lands Ecology Reserve } \\ \text { DOE } & \text { U.S. Department of Energy } \\ \text { DOH } & \text { Washington State Department of Health } \\ \text { DR } & \text { downriver } \\ \text { DWMP } & \text { Drinking Water Monitoring Project } \\ \text { FFTF } & \text { Fast Flux Test Facility } \\ \text { HEIS } & \text { Hanford Environmental Information System } \\ \text { HRM } & \text { Hanford River Marker } \\ \text { ICP-MS } & \text { inductively coupled plasma mass spectrometry } \\ \text { NASQAN } & \text { National Stream Quality Accounting Network } \\ \text { PNNL } & \text { Pacific Northwest National Laboratory } \\ \text { PRD } & \text { Priest Rivers Dam } \\ \text { SESP } & \text { Surface Environmental Surveillance Project } \\ \text { UR } & \text { upriver } \\ \text { USGS } & \text { U.S. Geological Survey }\end{array}$

\section{Frequency Symbols Used}

A

$\mathrm{BE}$

BW

$\mathrm{M}$

M Comp.

Q

Q Comp.

SA

TE annually

biennially (every 2 years)

biweekly (every 2 weeks)

monthly

monthly composite

quarterly

quarterly composite

semiannually (twice each year)

triennially (every 3 years)

\section{Analytical Symbols Used}

Generally, standard element, chemical, and isotope designations are used to indicate the analyses performed. Other analytical designations used include the following:

Alpha

Anions

Beta gross alpha activity of sample

major anions - generally chloride, fluoride, nitrate, nitrite, sulfate

gross beta activity of sample 
Gamma Scan

HTO

Hg-CVAA

$\mathrm{Hg}-\mathrm{CVAF}$

ICP-MS

Lo ${ }^{3} \mathrm{H}$

$\mathrm{Pu}$

TOC

$\mathrm{U}$

VOA analysis of photon energy spectrum for individual photon-emitting radionuclides tritiated water $\left({ }^{3} \mathrm{H}^{1} \mathrm{H}^{16} \mathrm{O}\right)$

mercury by cold vapor atomic absorbance spectrometry

total mercury in water by cold vapor atomic fluorescence

major metals by inductively coupled plasma mass spectrometry - samples unfiltered unless otherwise noted

low-level method for the electrolytic enrichment of tritium

isotopic plutonium $\left({ }^{238} \mathrm{Pu},{ }^{239 / 240} \mathrm{Pu}\right)$

total organic carbon

isotopic uranium $\left({ }^{234} \mathrm{U},{ }^{235} \mathrm{U},{ }^{238} \mathrm{U}\right)$

volatile organic compounds 


\section{Contents}

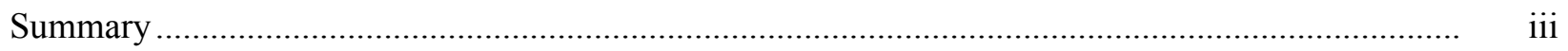

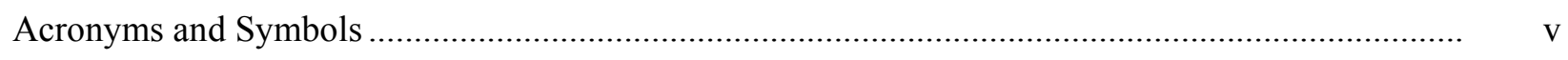

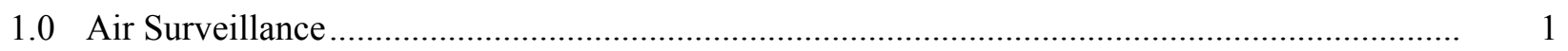

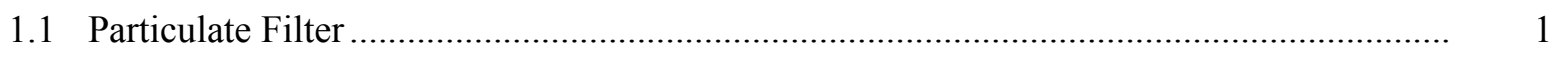

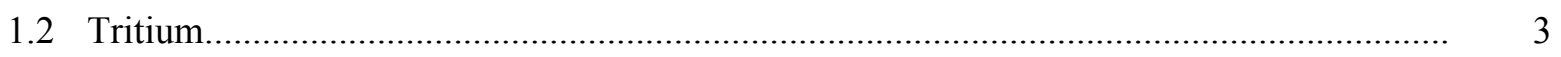

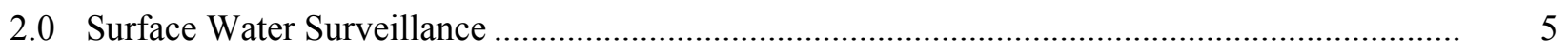

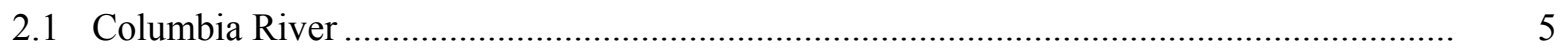

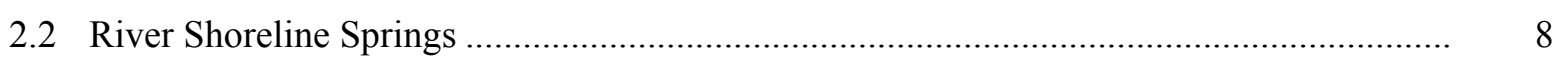

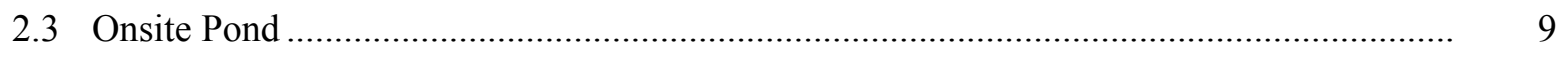

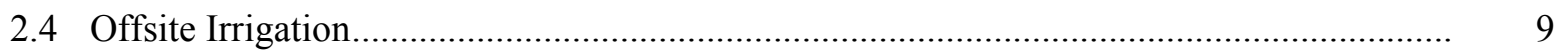

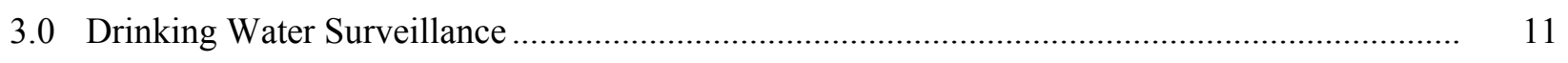

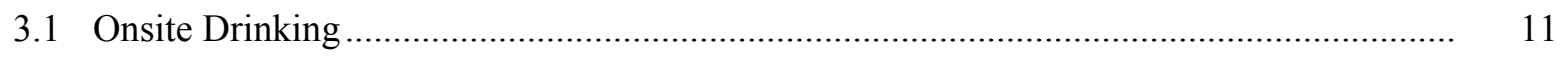

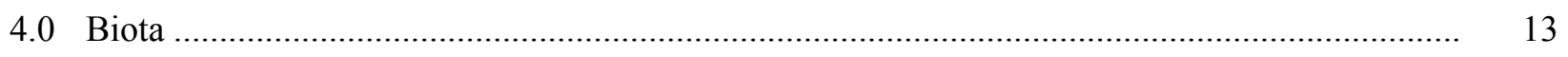

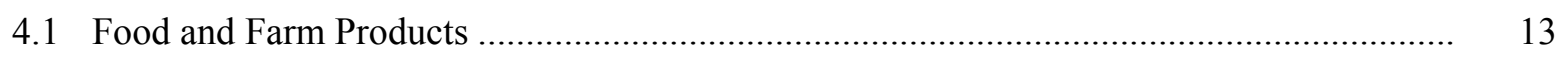

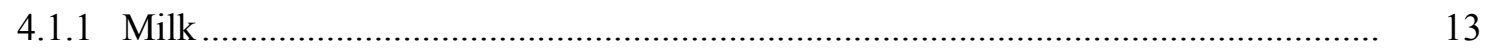

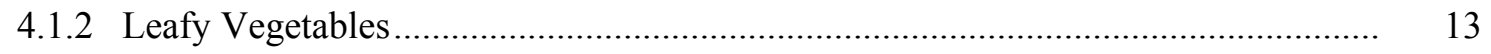

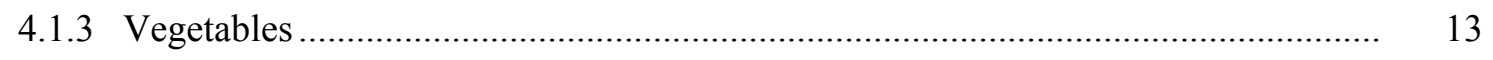

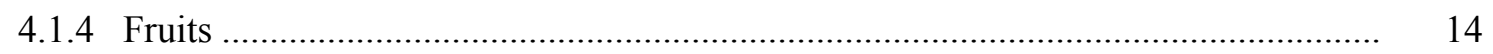

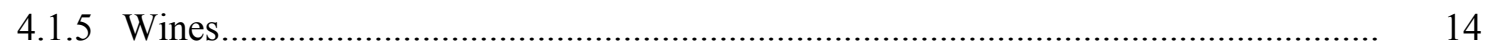

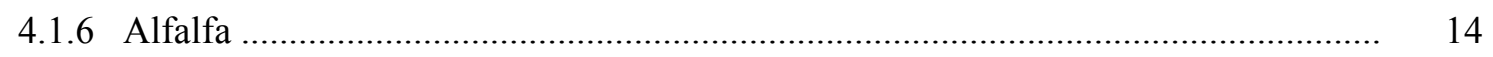

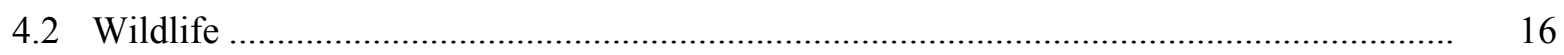

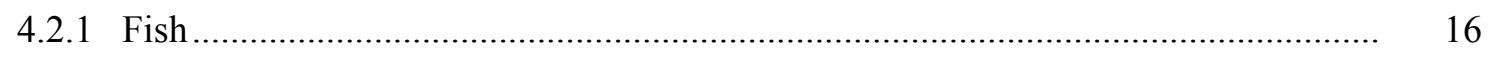

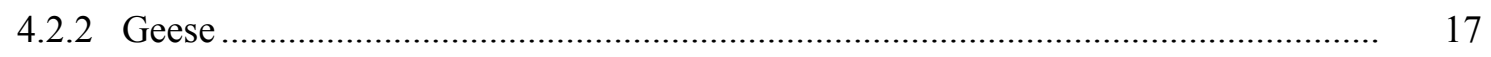

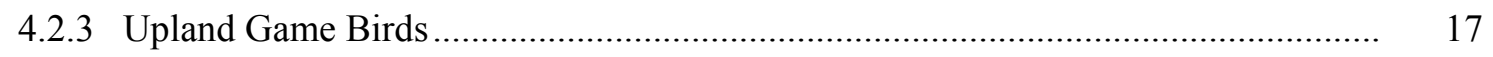

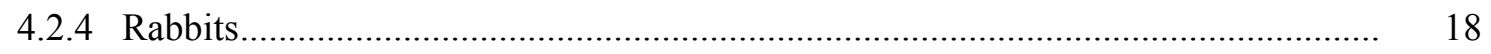

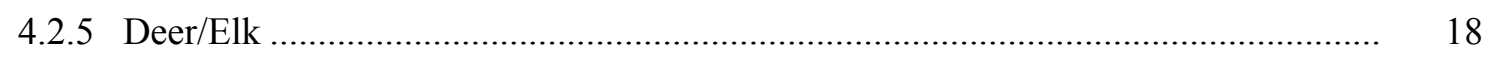

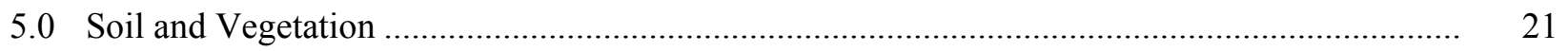

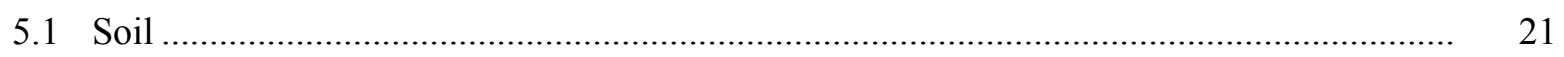

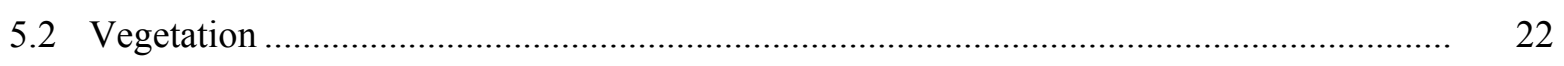

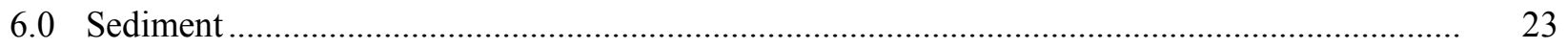

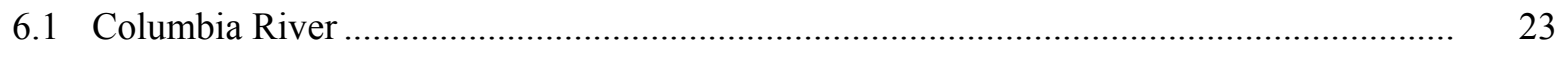

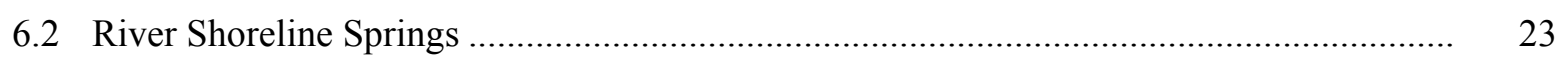

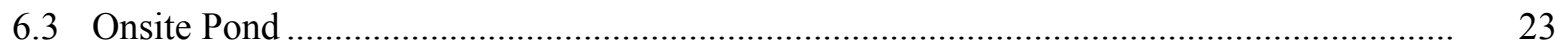




\section{Figures}

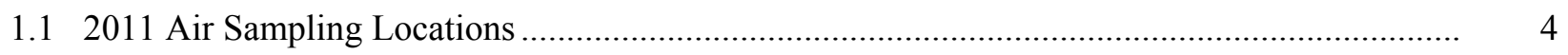

2.12011 Surface Water and Drinking Water Sampling Locations................................................ 10

4.12011 Food and Farm Products Sampling Locations ............................................................... 15

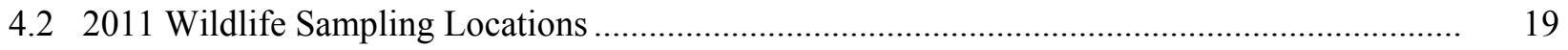

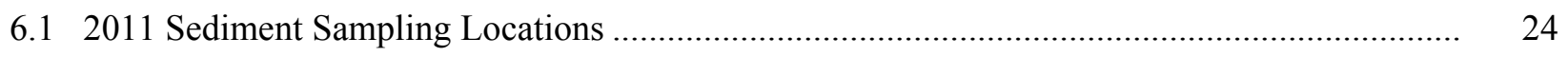




\subsection{Air Surveillance}

\subsection{Particulate Filter}

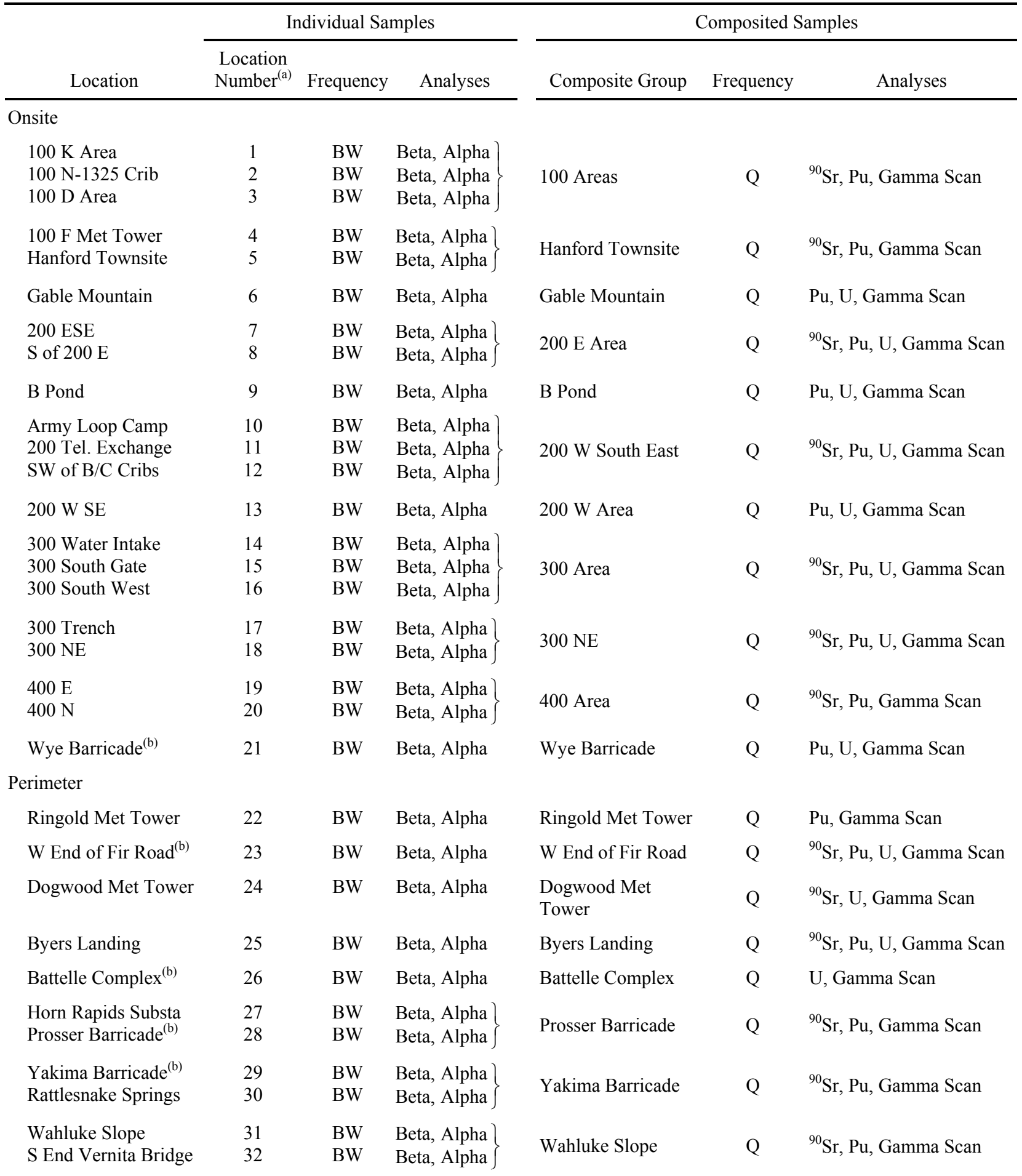




\section{Particulate Filter (contd)}

\begin{tabular}{|c|c|c|c|c|c|c|}
\hline \multirow[b]{2}{*}{ Location } & \multicolumn{3}{|c|}{ Individual Samples } & \multicolumn{3}{|c|}{ Composited Samples } \\
\hline & $\begin{array}{l}\text { Location } \\
\text { Number }^{(a)}\end{array}$ & Frequency & Analyses & Composite Group & Frequency & Analyses \\
\hline \multicolumn{7}{|l|}{ Community } \\
\hline Basin City School & 33 & BW & Beta, Alpha & Basin City School & Q & $\mathrm{Pu}, \mathrm{U}$, Gamma Scan \\
\hline Leslie Groves-Rchlnd & 34 & BW & Beta, Alpha & $\begin{array}{l}\text { Leslie Groves- } \\
\text { Rchlnd }\end{array}$ & Q & ${ }^{90} \mathrm{Sr}, \mathrm{Pu}, \mathrm{U}$, Gamma Scan \\
\hline $\begin{array}{l}\text { Pasco } \\
\text { Kennewick-Ely Street }\end{array}$ & $\begin{array}{l}35 \\
36\end{array}$ & $\begin{array}{l}\text { BW } \\
\text { BW }\end{array}$ & $\left.\begin{array}{l}\text { Beta } \\
\text { Beta, Alpha }\end{array}\right\}$ & Tri Cities & Q & ${ }^{90} \mathrm{Sr}, \mathrm{Pu}, \mathrm{U}, \mathrm{Gamma}$ Scan \\
\hline Benton City & 37 & BW & Beta & Benton City & Q & Gamma Scan \\
\hline Mattawa & 38 & BW & Beta & Mattawa & Q & Gamma Scan \\
\hline Othello & 39 & BW & Beta & Othello & Q & U, Gamma Scan \\
\hline \multicolumn{7}{|l|}{ Distant } \\
\hline Yakima & 40 & $\mathrm{BW}$ & Beta, Alpha & Yakima & Q & ${ }^{90} \mathrm{Sr}, \mathrm{Pu}, \mathrm{U}$, Gamma Scan \\
\hline $\begin{array}{l}\text { (a) Refer to Figure 1.1, } \\
\text { (b) Washington State De }\end{array}$ & '2011 Air S & Health (DO & $\begin{array}{l}\text { cations." } \\
\text { H) air sample }\end{array}$ & at this location. & & \\
\hline
\end{tabular}


1.2 Tritium

\begin{tabular}{|c|c|c|c|}
\hline Location & $\begin{array}{l}\text { Location } \\
\text { Number }^{(a)}\end{array}$ & Frequency & Analysis $^{(b)}$ \\
\hline \multicolumn{4}{|l|}{ Onsite } \\
\hline $100 \mathrm{~K}$ Area & 1 & M & ${ }^{3} \mathrm{H}$ \\
\hline $100 \mathrm{~N}-1325 \mathrm{Crib}$ & 2 & M & ${ }^{3} \mathrm{H}$ \\
\hline $200 \mathrm{ESE}$ & 7 & M & ${ }^{3} \mathrm{H}$ \\
\hline 200 Tel. Exchange & 11 & M & ${ }^{3} \mathrm{H}$ \\
\hline 300 Water Intake ${ }^{(c)}$ & 14 & M & ${ }^{3} \mathrm{H}$ \\
\hline 300 South Gate ${ }^{(\mathrm{d})}$ & 15 & M & ${ }^{3} \mathrm{H}$ \\
\hline 300 South West & 16 & M & ${ }^{3} \mathrm{H}$ \\
\hline 300 Trench & 17 & M & ${ }^{3} \mathrm{H}$ \\
\hline $300 \mathrm{NE}$ & 18 & M & ${ }^{3} \mathrm{H}$ \\
\hline $400 \mathrm{E}$ & 19 & M & ${ }^{3} \mathrm{H}$ \\
\hline \multicolumn{4}{|l|}{ Perimeter } \\
\hline Ringold Met Tower & 22 & M & ${ }^{3} \mathrm{H}$ \\
\hline W End of Fir Road & 23 & M & ${ }^{3} \mathrm{H}$ \\
\hline Dogwood Met Tower & 24 & M & ${ }^{3} \mathrm{H}$ \\
\hline Byers Landing & 25 & M & ${ }^{3} \mathrm{H}$ \\
\hline Battelle Complex $^{(\mathrm{c})}$ & 26 & M & ${ }^{3} \mathrm{H}$ \\
\hline Prosser Barricade & 28 & M & ${ }^{3} \mathrm{H}$ \\
\hline Wahluke Slope & 31 & M & ${ }^{3} \mathrm{H}$ \\
\hline \multicolumn{4}{|l|}{ Community } \\
\hline Basin City School & 33 & M & ${ }^{3} \mathrm{H}$ \\
\hline Leslie Groves-Rchlnd & 34 & M & ${ }^{3} \mathrm{H}$ \\
\hline \multicolumn{4}{|l|}{ Distant } \\
\hline Yakima & 40 & $\mathrm{M}$ & ${ }^{3} \mathrm{H}$ \\
\hline $\begin{array}{l}\text { (a) Refer to Figure 1.1, } \\
\text { (b) As tritiated water (H } \\
\text { (c) DOH air sampler als } \\
\text { (d) Two tritium samples }\end{array}$ & $\begin{array}{l}\text { Air Sampling } \\
\text { is location. } \\
\text { llected from }\end{array}$ & ocation. & \\
\hline
\end{tabular}




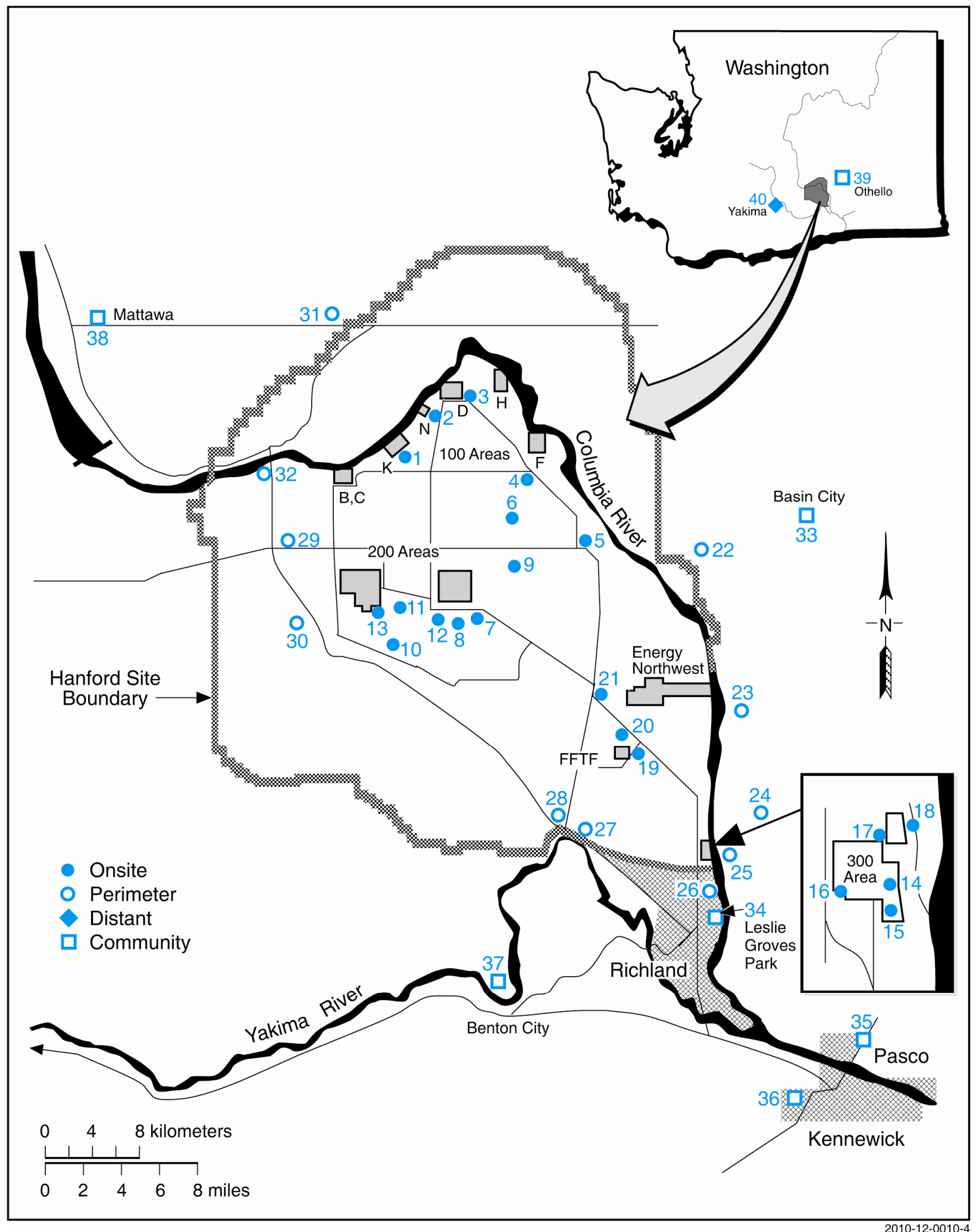

Figure 1.1. 2011 Air Sampling Locations 


\subsection{Surface Water Surveillance}

\subsection{Columbia River}

\begin{tabular}{|c|c|c|c|}
\hline Location $^{(a)}$ & Sample Type & Frequency & Analyses/Agency \\
\hline \multirow[t]{5}{*}{ Priest Rapids-River } & Cumulative & M Comp. $^{(\mathrm{b})}$ & Alpha, Beta, $\mathrm{Lo}^{3} \mathrm{H},{ }^{90} \mathrm{Sr},{ }^{99} \mathrm{Tc}, \mathrm{U} / \mathrm{DOH}^{(\mathrm{c})}$ \\
\hline & Particulate (filter) & M Comp. ${ }^{(d)}$ & Gamma Scan \\
\hline & & Q Comp. ${ }^{(\mathrm{d})}$ & $\mathrm{Pu}$ \\
\hline & Soluble (resin) & M Comp. $^{(d)}$ & Gamma Scan \\
\hline & & Q Comp. ${ }^{(\mathrm{d})}$ & $\mathrm{Pu}$ \\
\hline \multirow[t]{6}{*}{ Rich.Pmphs HRM 46.4} & Cumulative & M Comp..$^{(b)}$ & Alpha, Beta, Lo ${ }^{3} \mathrm{H},{ }^{90} \mathrm{Sr},{ }^{99} \mathrm{Tc}, \mathrm{U}$ \\
\hline & Particulate (filter) & M Comp. ${ }^{(\mathrm{d})}$ & Gamma Scan \\
\hline & & Q Comp. ${ }^{(\mathrm{d})}$ & $\mathrm{Pu}$ \\
\hline & Soluble (resin) & M Comp. $^{(d)}$ & Gamma Scan \\
\hline & & Q Comp. ${ }^{(\mathrm{d})}$ & $\mathrm{Pu}$ \\
\hline & Grab & $3 / \mathrm{Yr}$ & USGS-NASQAN $^{(\mathrm{e})}$ \\
\hline \multirow[t]{2}{*}{ Rich.Pmphs-1 HRM46.4 ${ }^{(\mathrm{f})}$} & Transect $^{(\mathrm{g})}$ & Q & Lo ${ }^{3} \mathrm{H},{ }^{90} \mathrm{Sr}, \mathrm{U}$, Anions \\
\hline & & A & ICP-MS, Hg-CVAF, ICP-MS Filtered, VOA \\
\hline \multirow[t]{2}{*}{ Rich.Pmphs-2 HRM46.4 } & Transect $^{(\mathrm{g})}$ & Q & Lo ${ }^{3} \mathrm{H},{ }^{90} \mathrm{Sr}, \mathrm{U}$, Anions \\
\hline & & A & ICP-MS, Hg-CVAF, ICP-MS Filtered, VOA \\
\hline \multirow[t]{2}{*}{ Rich.Pmphs-3 HRM46.4 } & Transect $^{(\mathrm{g})}$ & Q & Lo ${ }^{3} \mathrm{H},{ }^{90} \mathrm{Sr}, \mathrm{U}$, Anions \\
\hline & & A & ICP-MS, Hg-CVAF, ICP-MS Filtered, VOA \\
\hline \multirow[t]{2}{*}{ Rich.Pmphs-5 HRM46.4 } & Transect $^{(\mathrm{g})}$ & Q & Lo ${ }^{3} \mathrm{H},{ }^{90} \mathrm{Sr}, \mathrm{U}$, Anions \\
\hline & & A & ICP-MS, Hg-CVAF, ICP-MS Filtered, VOA \\
\hline \multirow[t]{2}{*}{ Rich.Pmphs-7 HRM46.4 } & Transect $^{(\mathrm{g})}$ & Q & Lo ${ }^{3} \mathrm{H},{ }^{90} \mathrm{Sr}, \mathrm{U}$, Anions \\
\hline & & A & ICP-MS, Hg-CVAF, ICP-MS Filtered, VOA \\
\hline \multirow[t]{2}{*}{ Rich.Pmphs-10 HRM46.4 } & Transect $^{(\mathrm{g})}$ & Q & Lo ${ }^{3} \mathrm{H},{ }^{90} \mathrm{Sr}, \mathrm{U}$, Anions \\
\hline & & $\mathrm{A}$ & ICP-MS, Hg-CVAF, ICP-MS Filtered, VOA \\
\hline \multirow[t]{2}{*}{ Rich.Pmphs HRM 43.5} & Transect $^{(\mathrm{g})}$ & Q & Lo ${ }^{3} \mathrm{H},{ }^{90} \mathrm{Sr}, \mathrm{U}$, Anions \\
\hline & & $\mathrm{A}$ & ICP-MS, Hg-CVAF, ICP-MS Filtered \\
\hline \multirow[t]{2}{*}{ Rich.Pmphs HRM 43.9} & Transect $^{(\mathrm{g})}$ & Q & Lo ${ }^{3} \mathrm{H},{ }^{90} \mathrm{Sr}, \mathrm{U}$, Anions \\
\hline & & A & ICP-MS, Hg-CVAF, ICP-MS Filtered \\
\hline \multirow[t]{2}{*}{ Rich.Pmphs HRM 45.0} & Transect $^{(\mathrm{g})}$ & Q & Lo ${ }^{3} \mathrm{H},{ }^{90} \mathrm{Sr}, \mathrm{U}$, Anions \\
\hline & & A & ICP-MS, Hg-CVAF, ICP-MS Filtered \\
\hline \multirow[t]{2}{*}{ Rich.Pmphs HRM 45.8} & Transect $^{(\mathrm{g})}$ & Q & Lo ${ }^{3} \mathrm{H},{ }^{90} \mathrm{Sr}, \mathrm{U}$, Anions \\
\hline & & A & ICP-MS, Hg-CVAF, ICP-MS Filtered \\
\hline Vernita & Grab & $3 / \mathrm{Yr}$ & USGS-NASQAN $^{(\mathrm{e})}$ \\
\hline \multirow[t]{2}{*}{ Vernita-1 HRM 0.3} & Transect $^{(\mathrm{g})}$ & Q & Lo ${ }^{3} \mathrm{H},{ }^{90} \mathrm{Sr}, \mathrm{U}$, Anions \\
\hline & & A & ${ }^{99} \mathrm{Tc}$, ICP-MS, Hg-CVAF, ICP-MS Filtered, VOA \\
\hline \multirow[t]{2}{*}{ Vernita-2 HRM 0.3} & Transect $^{(\mathrm{g})}$ & Q & Lo ${ }^{3} \mathrm{H},{ }^{90} \mathrm{Sr}, \mathrm{U}$, Anions \\
\hline & & A & ${ }^{99} \mathrm{Tc}, \mathrm{ICP}-\mathrm{MS}, \mathrm{Hg}-\mathrm{CVAF}$, ICP-MS Filtered, VOA \\
\hline \multirow[t]{2}{*}{ Vernita-3 HRM 0.3} & Transect $^{(\mathrm{g})}$ & Q & Lo ${ }^{3} \mathrm{H},{ }^{90} \mathrm{Sr}, \mathrm{U}$, Anions \\
\hline & & A & ${ }^{99} \mathrm{Tc}$, ICP-MS, Hg-CVAF, ICP-MS Filtered, VOA \\
\hline \multirow[t]{2}{*}{ Vernita-4 HRM 0.3} & Transect $^{(\mathrm{g})}$ & Q & Lo ${ }^{3} \mathrm{H},{ }^{90} \mathrm{Sr}, \mathrm{U}$, Anions \\
\hline & & A & ${ }^{99} \mathrm{Tc}, \mathrm{ICP}-\mathrm{MS}, \mathrm{Hg}-\mathrm{CVAF}$, ICP-MS Filtered, VOA \\
\hline
\end{tabular}




\section{Columbia River (contd)}

\begin{tabular}{|c|c|c|c|}
\hline Location $^{(a)}$ & Sample Type & Frequency & Analyses/Agency \\
\hline $100 \mathrm{~N}-1$ HRM $9.5^{(\mathrm{h})}$ & Transect $^{(\mathrm{g})}$ & A & Lo ${ }^{3} \mathrm{H},{ }^{90} \mathrm{Sr}, \mathrm{U}, \mathrm{ICP}-\mathrm{MS}$, ICP-MS Filtered, Anions \\
\hline $100 \mathrm{~N}-2$ HRM 9.5 & Transect $^{(\mathrm{g})}$ & A & Lo ${ }^{3} \mathrm{H},{ }^{90} \mathrm{Sr}, \mathrm{U}$, ICP-MS, ICP-MS Filtered, Anions \\
\hline $100 \mathrm{~N}-3$ HRM 9.5 & Transect $^{(\mathrm{g})}$ & A & Lo ${ }^{3} \mathrm{H},{ }^{90} \mathrm{Sr}, \mathrm{U}$, ICP-MS, ICP-MS Filtered, Anions \\
\hline $100 \mathrm{~N}-5$ HRM 9.5 & Transect $^{(\mathrm{g})}$ & A & Lo ${ }^{3} \mathrm{H},{ }^{90} \mathrm{Sr}$, U, ICP-MS, ICP-MS Filtered, Anions \\
\hline $100 \mathrm{~N}-7$ HRM 9.5 & Transect $^{(\mathrm{g})}$ & A & Lo ${ }^{3} \mathrm{H},{ }^{90} \mathrm{Sr}, \mathrm{U}$, ICP-MS, ICP-MS Filtered, Anions \\
\hline $100 \mathrm{~N}-10$ HRM 9.5 & Transect $^{(\mathrm{g})}$ & A & Lo ${ }^{3} \mathrm{H},{ }^{90} \mathrm{Sr}, \mathrm{U}$, ICP-MS, ICP-MS Filtered, Anions \\
\hline 100 N Shore HRM 8.4 & Transect ${ }^{(\mathrm{g})}$ & A & $\begin{array}{l}\text { Lo }{ }^{3} \mathrm{H},{ }^{90} \mathrm{Sr}, \mathrm{U}, \mathrm{ICP}-\mathrm{MS}, \mathrm{ICP}-\mathrm{MS} \text { Filtered, } \\
\text { Anions/DOH }{ }^{(\mathrm{i})}\end{array}$ \\
\hline 100 N Shore HRM 8.9 & Transect $^{(\mathrm{g})}$ & A & $\begin{array}{l}\text { Lo }{ }^{3} \mathrm{H},{ }^{90} \mathrm{Sr}, \mathrm{U}, \mathrm{ICP}-\mathrm{MS}, \text { ICP-MS Filtered, } \\
\text { Anions/DOH }{ }^{(\mathrm{i})}\end{array}$ \\
\hline 100 N Shore HRM 9.2 & Transect $^{(\mathrm{g})}$ & A & $\begin{array}{l}\text { Lo }{ }^{3} \mathrm{H},{ }^{90} \mathrm{Sr}, \mathrm{U}, \mathrm{ICP}-\mathrm{MS}, \text { ICP-MS Filtered, } \\
\text { Anions/DOH }{ }^{(\mathrm{i})}\end{array}$ \\
\hline 100 N Shore HRM 9.8 & Transect $^{(g)}$ & A & $\begin{array}{l}\text { Lo }{ }^{3} \mathrm{H},{ }^{90} \mathrm{Sr}, \mathrm{U}, \mathrm{ICP}-\mathrm{MS}, \text { ICP-MS Filtered, } \\
\text { Anions/DOH }{ }^{\mathrm{i})}\end{array}$ \\
\hline Hanfrd TS-1 HRM 28.7 & Transect $^{(\mathrm{g})}$ & A & $\begin{array}{l}\text { Lo }{ }^{3} \mathrm{H},{ }^{90} \mathrm{Sr},{ }^{99} \mathrm{Tc}, \mathrm{U}, \mathrm{ICP}-\mathrm{MS}, \mathrm{ICP}-\mathrm{MS} \text { Filtered, } \\
\text { Anions }\end{array}$ \\
\hline Hanfrd TS-2 HRM 28.7 & Transect $^{(\mathrm{g})}$ & A & Lo ${ }^{3} \mathrm{H},{ }^{90} \mathrm{Sr}$, U, ICP-MS, ICP-MS Filtered, Anions \\
\hline Hanfrd TS-3 HRM 28.7 & Transect $^{(\mathrm{g})}$ & A & Lo ${ }^{3} \mathrm{H},{ }^{90} \mathrm{Sr}$, U, ICP-MS, ICP-MS Filtered, Anions \\
\hline Hanfrd TS-5 HRM 28.7 & Transect $^{(\mathrm{g})}$ & A & Lo ${ }^{3} \mathrm{H},{ }^{90} \mathrm{Sr}, \mathrm{U}$, ICP-MS, ICP-MS Filtered, Anions \\
\hline Hanfrd TS-7 HRM 28.7 & Transect $^{(\mathrm{g})}$ & A & Lo ${ }^{3} \mathrm{H},{ }^{90} \mathrm{Sr}, \mathrm{U}$, ICP-MS, ICP-MS Filtered, Anions \\
\hline Hanfrd TS-10 HRM 28.7 & Transect $^{(\mathrm{g})}$ & A & Lo ${ }^{3} \mathrm{H},{ }^{90} \mathrm{Sr}, \mathrm{U}$, ICP-MS, ICP-MS Filtered, Anions \\
\hline Hanfrd Twnsite HRM26 & Transect $^{(\mathrm{g})}$ & A & $\begin{array}{l}\text { Lo }{ }^{3} \mathrm{H},{ }^{90} \mathrm{Sr},{ }^{99} \mathrm{Tc}, \mathrm{U}, \mathrm{ICP}-\mathrm{MS}, \mathrm{ICP}-\mathrm{MS} \text { Filtered, } \\
\text { Anions/DOH }{ }^{(\mathrm{i})}\end{array}$ \\
\hline Hanfrd Twnsite HRM27 & Transect $^{(\mathrm{g})}$ & A & $\begin{array}{l}\text { Lo }{ }^{3} \mathrm{H},{ }^{90} \mathrm{Sr},{ }^{99} \mathrm{Tc}, \mathrm{U}, \mathrm{ICP}-\mathrm{MS}, \mathrm{ICP}-\mathrm{MS} \text { Filtered, } \\
\text { Anions/DOH }{ }^{(\mathrm{i})}\end{array}$ \\
\hline Hanfrd Twnsite HRM28 & Transect $^{(g)}$ & A & $\begin{array}{l}\text { Lo }{ }^{3} \mathrm{H},{ }^{90} \mathrm{Sr},{ }^{99} \mathrm{Tc}, \mathrm{U}, \mathrm{ICP}-\mathrm{MS}, \mathrm{ICP}-\mathrm{MS} \text { Filtered, } \\
\text { Anions/DOH }{ }^{(\mathrm{i})}\end{array}$ \\
\hline Hanfrd Twnsite HRM30 & Transect $^{(\mathrm{g})}$ & A & $\begin{array}{l}\text { Lo }{ }^{3} \mathrm{H},{ }^{90} \mathrm{Sr},{ }^{99} \mathrm{Tc}, \mathrm{U}, \mathrm{ICP}-\mathrm{MS}, \mathrm{ICP}-\mathrm{MS} \text { Filtered, } \\
\text { Anions/DOH }{ }^{(\mathrm{i})}\end{array}$ \\
\hline 300 Area -1 HRM 43.1 & Transect $^{(\mathrm{g})}$ & A & Lo ${ }^{3} \mathrm{H},{ }^{90} \mathrm{Sr}$, U, ICP-MS, ICP-MS Filtered, Anions \\
\hline 300 Area -2 HRM 43.1 & Transect $^{(\mathrm{g})}$ & A & Lo ${ }^{3} \mathrm{H},{ }^{90} \mathrm{Sr}, \mathrm{U}$, ICP-MS, ICP-MS Filtered, Anions \\
\hline 300 Area -3 HRM 43.1 & Transect $^{(\mathrm{g})}$ & A & Lo ${ }^{3} \mathrm{H},{ }^{90} \mathrm{Sr}, \mathrm{U}$, ICP-MS, ICP-MS Filtered, Anions \\
\hline 300 Area -5 HRM 43.1 & Transect $^{(\mathrm{g})}$ & A & Lo ${ }^{3} \mathrm{H},{ }^{90} \mathrm{Sr}, \mathrm{U}$, ICP-MS, ICP-MS Filtered, Anions \\
\hline 300 Area -7 HRM 43.1 & Transect $^{(\mathrm{g})}$ & A & Lo ${ }^{3} \mathrm{H},{ }^{90} \mathrm{Sr}, \mathrm{U}$, ICP-MS, ICP-MS Filtered, Anions \\
\hline 300 Area -10 HRM 43.1 & Transect ${ }^{(\mathrm{g})}$ & A & Lo ${ }^{3} \mathrm{H},{ }^{90} \mathrm{Sr}$, U, ICP-MS, ICP-MS Filtered, Anions \\
\hline 300 Area Shr HRM41.5 & Transect $^{(\mathrm{g})}$ & $\mathrm{A}$ & $\begin{array}{l}\text { Lo }{ }^{3} \mathrm{H},{ }^{90} \mathrm{Sr}, \mathrm{U}, \mathrm{ICP}-\mathrm{MS}, \mathrm{ICP}-\mathrm{MS} \text { Filtered, } \\
\text { Anions/DOH }{ }^{(\mathrm{i})}\end{array}$ \\
\hline 300 Area Spring 42-2 & Transect $^{(\mathrm{g})}$ & A & $\begin{array}{l}\text { Lo }{ }^{3} \mathrm{H},{ }^{90} \mathrm{Sr}, \mathrm{U}, \mathrm{ICP}-\mathrm{MS}, \mathrm{ICP}-\mathrm{MS} \text { Filtered, } \\
\text { Anions/DOH }{ }^{(\mathrm{i})}\end{array}$ \\
\hline 300 Area Spr DR 42-2 & Transect $^{(\mathrm{g})}$ & A & $\begin{array}{l}\text { Lo }{ }^{3} \mathrm{H},{ }^{90} \mathrm{Sr}, \mathrm{U}, \mathrm{ICP}-\mathrm{MS}, \mathrm{ICP}-\mathrm{MS} \text { Filtered, } \\
\text { Anions/DOH }{ }^{(\mathrm{i})}\end{array}$ \\
\hline 300 Area Shr HRM42.9 & Transect $^{(\mathrm{g})}$ & A & $\begin{array}{l}\text { Lo }{ }^{3} \mathrm{H},{ }^{90} \mathrm{Sr}, \mathrm{U}, \mathrm{ICP}-\mathrm{MS}, \text { ICP-MS Filtered, } \\
\text { Anions/DOH }{ }^{(\mathrm{i})}\end{array}$ \\
\hline
\end{tabular}




\section{Columbia River (contd)}

\begin{tabular}{cccl}
\hline Location $^{(\mathrm{a})}$ & Sample Type & Frequency & \multicolumn{1}{c}{ Analyses/Agency } \\
\hline 300 Area Outfl13 & Grab & $\mathrm{Q}$ & Lo $^{3} \mathrm{H},{ }^{90} \mathrm{Sr}, \mathrm{U}$, Anions \\
& & A & ICP-MS, ICP-MS Filtered \\
\hline
\end{tabular}

(a) Refer to Figure 2.1, "2011 Surface Water and Drinking Water Sampling Locations." Hanford River Markers (HRMs) are a series of signposts along the Hanford Site shoreline of the Columbia River that are roughly $1.6 \mathrm{~km}$ (1 mi) apart. The Vernita Bridge is HRM \#0 and Ferry Street in Richland is HRM \#46. Samples collected between HRMs are assigned a decimal.

(b) Sample is collected weekly and composited monthly for analysis.

(c) Additional sample provided to the DOH (January and June only).

(d) Sample is collected biweekly and composited for analysis.

(e) Analyses are performed by the U.S. Geological Survey (USGS) in conjunction with the National Stream Quality Accounting Network (NASQAN) Program, and include conductance, $\mathrm{pH}$, temperature, turbidity, dissolved oxygen, hardness, $\mathrm{Ca}, \mathrm{Mg}$, alkalinity, carbonates, sulfate, $\mathrm{Cl}$, F, solids, $\mathrm{NH}_{4}-\mathrm{N}, \mathrm{NO}_{3}+\mathrm{NO}_{2}, \mathrm{~N}-\mathrm{Kjeldahl}, \mathrm{P}, \mathrm{Cr}, \mathrm{Fe}$, and dissolved organic carbon.

(f) Quality assurance sample submitted for analyses twice per year.

(g) Samples collected from cross-river transects and near-shore locations.

(h) Quality assurance sample submitted for analyses once per year.

(i) Additional sample provided to the $\mathrm{DOH}$. 


\subsection{River Shoreline Springs}

\begin{tabular}{|c|c|c|c|c|}
\hline Location $^{(a)}$ & $\mathrm{HRM}^{(\mathrm{b})}$ & $\begin{array}{l}\text { Sample } \\
\text { Type }\end{array}$ & $\begin{array}{c}\text { Fre- } \\
\text { quency }\end{array}$ & Analyses/Agency \\
\hline 100-B Spring 38-3 & 3.8 & Grab & A & $\begin{array}{l}\text { Alpha, Beta, }{ }^{3} \mathrm{H},{ }^{90} \mathrm{Sr},{ }^{99} \mathrm{Tc}, \text { Gamma Scan, ICP-MS, Hg-CVAF, } \\
\text { ICP-MS Filtered, Anions, VOA }\end{array}$ \\
\hline 100-B Spring 39-2 & 3.9 & Grab & A & $\begin{array}{l}\text { Alpha, Beta, }{ }^{3} \mathrm{H},{ }^{90} \mathrm{Sr},{ }^{99} \mathrm{Tc} \text {, Gamma Scan, ICP-MS, Hg-CVAF, } \\
\text { ICP-MS Filtered, Anions, VOA }\end{array}$ \\
\hline 100-K Spring 63-1 & 6.3 & Grab & A & $\begin{array}{l}\text { Alpha, Beta, }{ }^{3} \mathrm{H},{ }^{90} \mathrm{Sr}, \text { Gamma Scan, ICP-MS, Hg-CVAF, ICP-MS } \\
\text { Filtered, Anions, VOA/DOH }{ }^{(\mathrm{c})}\end{array}$ \\
\hline 100-K Spring 77-1 & 7.6 & Grab & A & $\begin{array}{l}\text { Alpha, Beta, }{ }^{3} \mathrm{H},{ }^{90} \mathrm{Sr}, \mathrm{Gamma} \text { Scan, ICP-MS, Hg-CVAF, ICP-MS } \\
\text { Filtered, Anions, VOA/DOH }{ }^{(\mathrm{c})}\end{array}$ \\
\hline $100-\mathrm{N}$ Spring 8-13 & 9.3 & Grab & A & $\begin{array}{l}\text { Alpha, Beta, }{ }^{3} \mathrm{H},{ }^{90} \mathrm{Sr} \text {, Gamma Scan, ICP-MS, Hg-CVAF, ICP-MS } \\
\text { Filtered, Anions/DOH }{ }^{(\mathrm{c})}\end{array}$ \\
\hline 100-D Spring 102-1 & 10.2 & Grab & A & $\begin{array}{l}\text { Alpha, Beta, }{ }^{3} \mathrm{H},{ }^{90} \mathrm{Sr} \text {, Gamma Scan, ICP-MS, Hg-CVAF, ICP-MS } \\
\text { Filtered, Anions/DOH }{ }^{(\mathrm{c})}\end{array}$ \\
\hline 100-D Spring 110-1 & 11.0 & Grab & A & $\begin{array}{l}\text { Alpha, Beta, }{ }^{3} \mathrm{H},{ }^{90} \mathrm{Sr} \text {, Gamma Scan, ICP-MS, Hg-CVAF, ICP-MS } \\
\text { Filtered, Anions } / \mathrm{DOH}^{(\mathrm{c})}\end{array}$ \\
\hline 100-H Spring 145-1 & 14.4 & Grab & A & $\begin{array}{l}\text { Alpha, Beta, }{ }^{3} \mathrm{H},{ }^{90} \mathrm{Sr},{ }^{99} \mathrm{Tc}, \mathrm{U}, \text { Gamma Scan, ICP-MS, Hg-CVAF, } \\
\text { ICP-MS Filtered, Anions }\end{array}$ \\
\hline 100-H Spring 153-1 & 15.3 & Grab & A & $\begin{array}{l}\text { Alpha, Beta, }{ }^{3} \mathrm{H},{ }^{90} \mathrm{Sr},{ }^{99} \mathrm{Tc}, \mathrm{U}, \text { Gamma Scan, ICP-MS, Hg-CVAF, } \\
\text { ICP-MS Filtered, Anions }\end{array}$ \\
\hline 100-F Spring 207-1 & 21.3 & Grab & A & $\begin{array}{l}\text { Alpha, Beta, }{ }^{3} \mathrm{H},{ }^{90} \mathrm{Sr}, \mathrm{U}, \text { Gamma Scan, ICP-MS, Hg-CVAF, } \\
\text { ICP-MS Filtered, Anions, VOA }\end{array}$ \\
\hline Hanford Spr UR 28-2 ${ }^{(\mathrm{d})}$ & 27.8 & Grab & A & $\begin{array}{l}\text { Alpha, Beta, }{ }^{3} \mathrm{H},{ }^{99} \mathrm{Tc}, \mathrm{U},{ }^{129} \mathrm{I}, \mathrm{Gamma} \text { Scan, ICP-MS, Hg-CVAF, } \\
\text { ICP-MS Filtered, Anions/DOH }{ }^{(\mathrm{c})}\end{array}$ \\
\hline Hanford Spring 28-2 & 28.1 & Grab & A & $\begin{array}{l}\text { Alpha, Beta, }{ }^{3} \mathrm{H},{ }^{99} \mathrm{Tc}, \mathrm{U},{ }^{129} \mathrm{I}, \text { Gamma Scan, ICP-MS, Hg-CVAF, } \\
\text { ICP-MS Filtered, Anions/DOH }{ }^{(\mathrm{c})}\end{array}$ \\
\hline Hanford Spr DR 28-2 ${ }^{(\mathrm{e})}$ & 28.3 & Grab & A & $\begin{array}{l}\text { Alpha, Beta, }{ }^{3} \mathrm{H},{ }^{99} \mathrm{Tc}, \mathrm{U},{ }^{129} \mathrm{I}, \text { Gamma Scan, ICP-MS, Hg-CVAF, } \\
\text { ICP-MS Filtered, Anions/DOH }{ }^{(\mathrm{c})}\end{array}$ \\
\hline 300 Area Spring 41-9 & 41.9 & Grab & A & $\begin{array}{l}\text { Alpha, Beta, }{ }^{3} \mathrm{H},{ }^{90} \mathrm{Sr}, \mathrm{U}, \text { Gamma Scan, ICP-MS, Hg-CVAF, } \\
\text { ICP-MS Filtered, Anions }\end{array}$ \\
\hline 300 Area Spring 42-2 & 42.1 & Grab & A & $\begin{array}{l}\text { Alpha, Beta, }{ }^{3} \mathrm{H},{ }^{90} \mathrm{Sr}, \mathrm{U},{ }^{129} \mathrm{I}, \mathrm{Gamma} \text { Scan, ICP-MS, Hg-CVAF, } \\
\text { ICP-MS Filtered, Anions, VOA/ DOH }{ }^{(\mathrm{c})}\end{array}$ \\
\hline 300 Area Spr DR 42-2 & 42.4 & Grab & A & $\begin{array}{l}\text { Alpha, Beta, }{ }^{3} \mathrm{H},{ }^{90} \mathrm{Sr}, \mathrm{U},{ }^{129} \mathrm{I}, \mathrm{Gamma} \text { Scan, ICP-MS, Hg-CVAF, } \\
\text { ICP-MS Filtered, Anions, VOA/ DOH }{ }^{(\mathrm{c})}\end{array}$ \\
\hline 300 Area Spring 42-7 & 42.7 & Grab & A & Alpha, Beta, ${ }^{3} \mathrm{H},{ }^{90} \mathrm{Sr}, \mathrm{U}$, Gamma Scan, Anions \\
\hline Richland Spr(SRL 437-1) & 43.7 & Grab & A & $\begin{array}{l}\text { Alpha, Beta, }{ }^{3} \mathrm{H},{ }^{90} \mathrm{Sr}, \mathrm{U}, \text { Gamma Scan, ICP-MS, Hg-CVAF, } \\
\text { ICP-MS Filtered, Anions }\end{array}$ \\
\hline \multirow{2}{*}{\multicolumn{5}{|c|}{$\begin{array}{l}\text { (a) Refer to Figure 2.1, "2011 Surface Water and Drinking Water Sampling Locations." } \\
\text { (b) HRMs are a series of signposts along the Hanford Site shoreline of the Columbia River that are roughly } 1.6 \mathrm{~km}(1 \mathrm{mi}) \text { apart. } \\
\text { The Vernita Bridge is HRM \#0 and Ferry Street in Richland is HRM \#46. Samples collected between HRMs are assigned a } \\
\text { decimal. }\end{array}$}} \\
\hline & & & & \\
\hline \multicolumn{5}{|c|}{ (c) Additional sample provided to the DOH. } \\
\hline \multicolumn{5}{|c|}{ (d) $\mathrm{UR}=$ Upriver from noted location. } \\
\hline $\mathrm{DR}=$ Downriver fron & noted loca & & & \\
\hline
\end{tabular}




\subsection{Onsite Pond}

\begin{tabular}{|c|c|c|c|}
\hline Location $^{(a)}$ & Sample Type & Frequency & Analyses \\
\hline West Lake & Grab & $\mathrm{Q}$ & ${ }^{3} \mathrm{H}$ \\
\hline FFTF Pond $^{(b)}$ & Grab & Q & Alpha, Beta, ${ }^{3} \mathrm{H}$, Gamma Scan \\
\hline
\end{tabular}

(a) Refer to Figure 2.1, "2011 Surface Water and Drinking Water Sampling Locations."

(b) Quality assurance sample submitted for analyses once per year. FFTF = Fast Flux Test Facility (400 Area).

\subsection{Offsite Irrigation}

\begin{tabular}{cccc}
\hline \multicolumn{1}{c}{ Location $^{(\mathrm{a})}$} & Sample Type & Frequency & Analyses/Agency \\
\hline Riverview Canal & Grab & 3 (May-Sept) & Alpha, Beta, Lo ${ }^{3} \mathrm{H},{ }^{90} \mathrm{Sr}, \mathrm{U}, \mathrm{Gamma}$ Scan/DOH \\
Horn Rapids Area & Grab & 3 (May-Sept) & Alpha, Beta, Lo ${ }^{3} \mathrm{H},{ }^{90} \mathrm{Sr}, \mathrm{U}, \mathrm{Gamma} \mathrm{Scan} / \mathrm{DOH}^{(\mathrm{b})}$ \\
\hline
\end{tabular}

(a) Refer to Figure 2.1, "2011 Surface Water and Drinking Water Sampling Locations."

(b) Additional sample provided to the $\mathrm{DOH}$. 


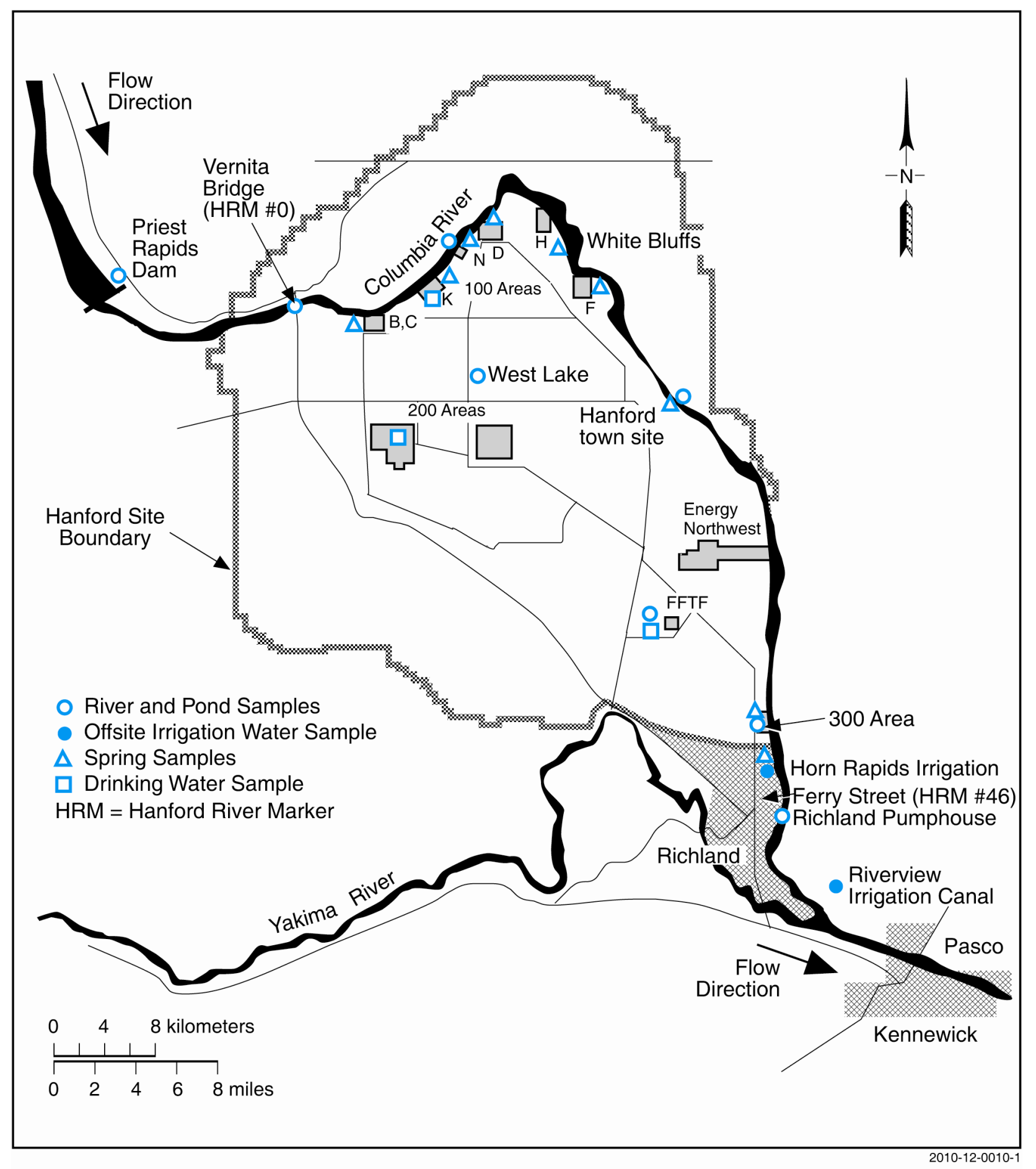

Figure 2.1. 2011 Surface Water and Drinking Water Sampling Locations 


\subsection{Drinking Water Surveillance}

\subsection{Onsite Drinking}

\begin{tabular}{|c|c|c|c|c|c|c|}
\hline \multirow[b]{2}{*}{ Location $^{\text {(a) }}$} & \multirow[b]{2}{*}{$\begin{array}{c}\text { Sample } \\
\text { Type }\end{array}$} & \multicolumn{2}{|c|}{ Individual Samples } & \multicolumn{3}{|c|}{ Composited Samples } \\
\hline & & $\begin{array}{c}\text { Fre- } \\
\text { quency }\end{array}$ & Analyses/Agency & Composite Group & $\begin{array}{c}\text { Fre- } \\
\text { quency }\end{array}$ & Analyses/Agency \\
\hline $200 \mathrm{~W}$ Area & Grab & $\mathrm{M}^{(\mathrm{b})}$ & & $200 \mathrm{~W}$ Area & $\mathrm{Q}$ & Beta \\
\hline $100 \mathrm{~K}$ Area & Grab & $\mathrm{M}^{(\mathrm{b})}$ & & $100 \mathrm{~K}$ Area & Q & Beta \\
\hline 400 Area Well P-14 & Grab & $\mathrm{M}^{(\mathrm{b})}$ & & 400 Area Well P-14 & Q & Beta \\
\hline 400 Area & Grab & $\mathrm{M}^{(\mathrm{b})}$ & & 400 Area & Q & Beta \\
\hline 200 W Area & Grab & $\mathrm{Q}^{(\mathrm{c})}$ & Alpha $\}$ & $200 \mathrm{~W}$ Area & A & ${ }^{90} \mathrm{Sr},{ }^{3} \mathrm{H}$ \\
\hline $100 \mathrm{~K}$ Area & Grab & $\mathrm{Q}^{(\mathrm{c})}$ & Alpha $\}$ & $100 \mathrm{~K}$ Area & A & ${ }^{90} \mathrm{Sr},{ }^{3} \mathrm{H}$ \\
\hline 400 Area Well P-14 & Grab & $\mathrm{Q}^{(\mathrm{c})}$ & Alpha $\}$ & 400 Area Well P-14 & A & ${ }^{90} \mathrm{Sr},{ }^{3} \mathrm{H}$ \\
\hline 400 Area & Grab & $\mathrm{Q}^{(\mathrm{c})}$ & Alpha, $\left.{ }^{3} \mathrm{H} / \mathrm{DOH}^{(\mathrm{d})}\right\}$ & 400 Area & A & ${ }^{90} \mathrm{Sr} / \mathrm{DOH}^{(\mathrm{e})}$ \\
\hline \multicolumn{7}{|c|}{ (a) Refer to Figure 2.1, "2011 Surface Water and Drinking Water Sampling Locations." } \\
\hline \multicolumn{7}{|c|}{ (b) Sample is collected monthly and composited for quarterly analysis. } \\
\hline \multicolumn{7}{|c|}{ (c) Sample is collected and analyzed quarterly and then composited for annual analysis. } \\
\hline \multicolumn{7}{|c|}{ (d) Additional sample provided to the $\mathrm{DOH}$ for ${ }^{3} \mathrm{H}$ analysis (January only). } \\
\hline \multicolumn{7}{|c|}{ (e) Additional sample provided quarterly to the $\mathrm{DOH}$ for annual ${ }^{90} \mathrm{Sr}$ analysis. } \\
\hline
\end{tabular}




\subsection{Biota}

\subsection{Food and Farm Products}

\subsubsection{Milk}

\begin{tabular}{|c|c|c|}
\hline Location $^{(\mathrm{a})}$ & Frequency & Analyses \\
\hline East Wahluke Area $^{(b)}$ & Q & Lo ${ }^{3} \mathrm{H},{ }^{90} \mathrm{Sr}$, Gamma Scan \\
\hline Sagemoor Composite ${ }^{(b, c)}$ & Q & Lo ${ }^{3} \mathrm{H},{ }^{90} \mathrm{Sr}$, Gamma Scan \\
\hline Sunnyside Area & Q & Lo ${ }^{3} \mathrm{H},{ }^{90} \mathrm{Sr}$, Gamma Scan \\
\hline
\end{tabular}

(a) Refer to Figure 4.1, "2011 Food and Farm Products Sampling Locations."

(b) Sample composited from multiple dairies in each area.

(c) Quality assurance sample submitted for analyses once per year.

\subsubsection{Leafy Vegetables}

\begin{tabular}{lcc}
\hline \multicolumn{1}{c}{ Location $^{(\mathrm{a}, \mathrm{b})}$} & Frequency $^{(\mathrm{c})}$ & \multicolumn{1}{c}{ Analyses/Agency } \\
\hline Riverview Area & A & ${ }^{90} \mathrm{Sr}$, Gamma Scan/DOH \\
Sunnyside Area & A & ${ }^{90} \mathrm{Sr}$, Gamma Scan \\
Sagemoor Area & BE $(2011)$ & ${ }^{90} \mathrm{Sr}$, Gamma Scan/DOH \\
East Wahluke Area & BE (2012) & ${ }^{90} \mathrm{Sr}$, Gamma Scan/DOH \\
\hline
\end{tabular}

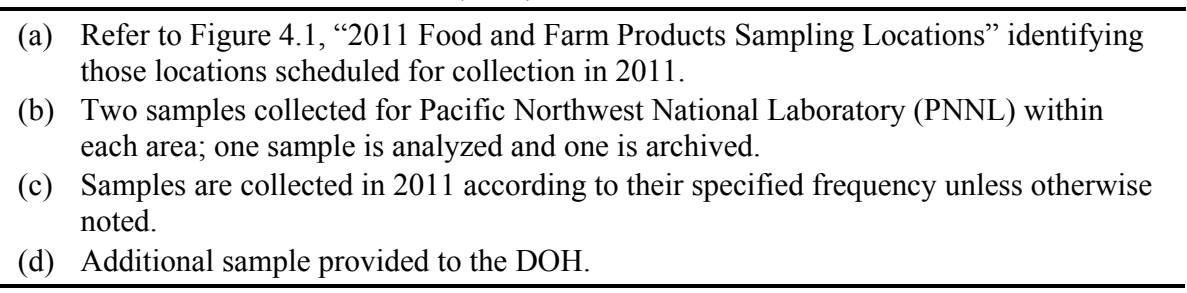

\subsubsection{Vegetables}

\begin{tabular}{|c|c|c|c|}
\hline Location $^{(\mathrm{a}, \mathrm{b})}$ & Sample Type & Frequency $^{(\mathrm{c})}$ & Analyses/Agency \\
\hline Riverview Area ${ }^{(\mathrm{d})}$ & Potatoes & A & ${ }^{90} \mathrm{Sr}$, Gamma Scan/DOH ${ }^{(\mathrm{e})}$ \\
\hline Sunnyside Area & Potatoes & A & ${ }^{90} \mathrm{Sr}$, Gamma Scan \\
\hline East Wahluke Area & Potatoes & A & ${ }^{90} \mathrm{Sr}$, Gamma Scan/DOH ${ }^{(\mathrm{e})}$ \\
\hline Horn Rapids Area & Potatoes & BE (2011) & ${ }^{90} \mathrm{Sr}$, Gamma Scan/DOH ${ }^{(\mathrm{e})}$ \\
\hline Sagemoor Area & Potatoes & TE (2012) & ${ }^{90} \mathrm{Sr}$, Gamma Scan/DOH ${ }^{(\mathrm{e})}$ \\
\hline
\end{tabular}

(a) Refer to Figure 4.1, "2011 Food and Farm Products Sampling Locations" identifying those locations scheduled for collection in 2011.

(b) Two samples collected for PNNL within each area; one sample is analyzed and one is archived.

(c) Samples are collected in 2011 according to their specified frequency unless otherwise noted.

(d) Other vegetables may be substituted if potatoes are not available.

(e) Additional sample provided to the $\mathrm{DOH}$. 


\subsubsection{Fruits}

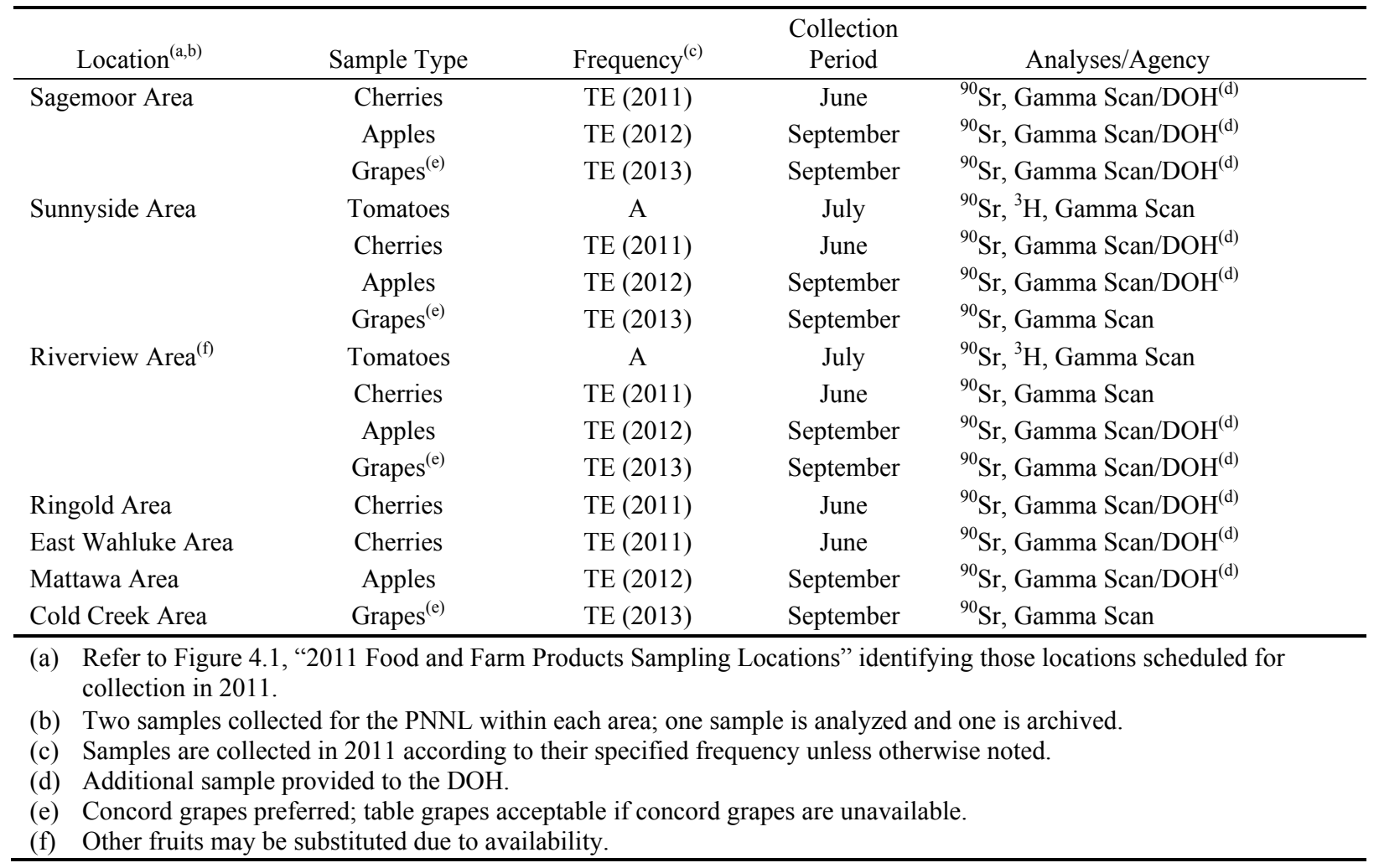

\subsubsection{Wines}

\begin{tabular}{|c|c|c|c|c|}
\hline Location $^{(\mathrm{a}, \mathrm{b})}$ & Sample Type & Frequency & $\begin{array}{l}\text { Collection } \\
\text { Period }\end{array}$ & Analyses/Agency \\
\hline \multirow[t]{2}{*}{ Columbia Basin $^{(\mathrm{c})}$} & White & $\mathrm{BE}(2011)$ & December & Lo $^{3} \mathrm{H}$, Gamma Scan/DOH ${ }^{(\mathrm{d})}$ \\
\hline & Red & BE (2011) & December & Lo ${ }^{3} \mathrm{H}$, Gamma Scan/DOH ${ }^{(\mathrm{d})}$ \\
\hline \multirow[t]{2}{*}{ Yakima Valley } & White & $\mathrm{BE}(2011)$ & December & Lo ${ }^{3} \mathrm{H}$, Gamma Scan/DOH ${ }^{(\mathrm{d})}$ \\
\hline & Red & BE (2011) & December & Lo ${ }^{3} \mathrm{H}$, Gamma Scan/DOH ${ }^{(\mathrm{d})}$ \\
\hline \multirow[t]{2}{*}{ Mattawa Area } & White & BE (2011) & December & Lo ${ }^{3} \mathrm{H}$, Gamma Scan/DOH ${ }^{(\mathrm{d})}$ \\
\hline & Red & BE (2011) & December & Lo ${ }^{3} \mathrm{H}$, Gamma Scan/DOH ${ }^{(\mathrm{d})}$ \\
\hline \multicolumn{5}{|c|}{$\begin{array}{l}\text { (a) Refer to Figure 4.1, "2011 Food and Farm Produc } \\
\text { (b) Two samples of each type collected for PNNL wi } \\
\text { (c) Location refers to Benton and Franklin Counties. } \\
\text { (d) Additional sample provided to the DOH. }\end{array}$} \\
\hline
\end{tabular}

\subsubsection{Alfalfa}

\begin{tabular}{lcccc}
\hline & & & Collection & \\
\multicolumn{1}{c}{ Location $^{(\mathrm{a}, \mathrm{b})}$} & Sample Type & Frequency & Period & \multicolumn{1}{c}{ Analyses/Agency } \\
\hline Sagemoor Area & Alfalfa & BE $(2011)$ & May & ${ }^{90} \mathrm{Sr}$, Gamma Scan \\
Riverview Area & Alfalfa & BE $(2011)$ & May & ${ }^{90} \mathrm{Sr}, \mathrm{Gamma} S c a n / \mathrm{DOH}^{(\mathrm{c})}$ \\
Sunnyside Area & Alfalfa & BE $(2011)$ & May & ${ }^{90} \mathrm{Sr}, \mathrm{Gamma}$ Scan \\
Horn Rapids Area & Alfalfa & BE (2011) & May & ${ }^{90} \mathrm{Sr}, \mathrm{Gamma} \mathrm{Scan} / \mathrm{DOH}^{(\mathrm{c})}$ \\
\hline
\end{tabular}

(a) Refer to Figure 4.1, "2011 Food and Farm Products Sampling Locations."

(b) Two samples collected for PNNL within each area; one sample is analyzed and one is archived.

(c) Additional sample provided to the $\mathrm{DOH}$. 


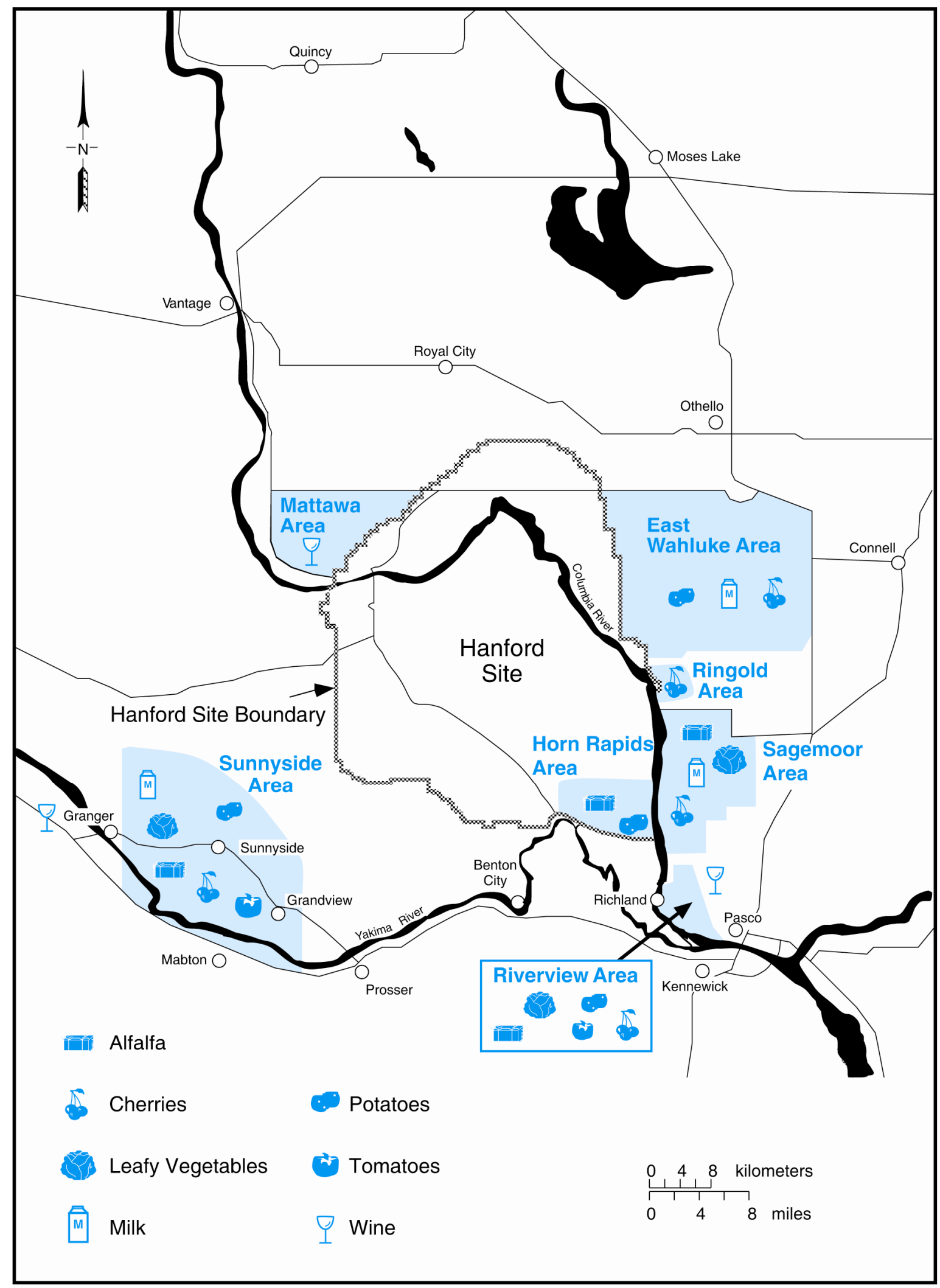

2010-12-0010-2

Figure 4.1. 2011 Food and Farm Products Sampling Locations 


\subsection{Wildlife}

\subsubsection{Fish}

\begin{tabular}{|c|c|c|c|c|c|}
\hline Location $^{(a)}$ & $\begin{array}{c}\text { Species/ } \\
\text { Sample }\end{array}$ & $\begin{array}{c}\text { Number } \\
\text { of Samples }\end{array}$ & Frequency $^{(\mathrm{b})}$ & $\begin{array}{l}\text { Collection } \\
\text { Period }\end{array}$ & Analyses/Agency \\
\hline \multirow[t]{4}{*}{$100 \mathrm{~F}$ Slough } & Bass & & & & \\
\hline & Fillet & 5 & TE (2011) & April-June & Gamma Scan/DOH ${ }^{(\mathrm{c})}$ \\
\hline & Carcass & 5 & TE (2011) & April-June & ${ }^{90} \mathrm{Sr} / \mathrm{DOH}^{(\mathrm{c})}$ \\
\hline & Liver $^{(\mathrm{d})}$ & 5 & TE (2011) & April-June & ICP-MS, Hg-CVAA \\
\hline \multirow[t]{4}{*}{ Hanford Slough } & Bass & & & & \\
\hline & Fillet & 5 & TE (2011) & April-June & Gamma Scan/DOH ${ }^{(\mathrm{c})}$ \\
\hline & Carcass & 5 & TE (2011) & April-June & ${ }^{90} \mathrm{Sr} / \mathrm{DOH}^{(\mathrm{c})}$ \\
\hline & Liver $^{(d)}$ & 5 & TE (2011) & April-June & ICP-MS, Hg-CVAA \\
\hline \multirow[t]{8}{*}{300 Area } & Bass & & & & \\
\hline & Fillet & 5 & TE (2011) & April-June & Gamma Scan, U/DOH ${ }^{(c)}$ \\
\hline & Carcass & 5 & TE (2011) & April-June & ${ }^{90} \mathrm{Sr} / \mathrm{DOH}^{(\mathrm{c})}$ \\
\hline & Liver $^{(\mathrm{d})}$ & 5 & TE (2011) & April-June & ICP-MS, Hg-CVAA \\
\hline & Carp & & & & \\
\hline & Fillet & 5 & BE (2012) & April-July & Gamma Scan, U/DOH ${ }^{(c)}$ \\
\hline & Carcass & 5 & BE (2012) & April-July & ${ }^{90} \mathrm{Sr} / \mathrm{DOH}^{(\mathrm{c})}$ \\
\hline & Liver $^{(d)}$ & 5 & BE (2012) & April-July & ICP-MS, Hg-CVAA \\
\hline \multicolumn{6}{|l|}{ Background - Desert } \\
\hline \multirow[t]{8}{*}{ Aire/Vantage } & Bass & & & & \\
\hline & Fillet & 5 & TE (2011) & April-June & Gamma Scan, U \\
\hline & Carcass & 5 & TE (2011) & April-June & ${ }^{90} \mathrm{Sr}$ \\
\hline & Liver $^{(\mathrm{d})}$ & 5 & TE (2011) & April-June & ICP-MS, Hg-CVAA \\
\hline & Carp & & & & \\
\hline & Fillet & 5 & BE (2012) & Oct-Nov & Gamma Scan, U/DOH $^{(\mathrm{c})}$ \\
\hline & Carcass & 5 & BE (2012) & Oct-Nov & ${ }^{90} \mathrm{Sr} / \mathrm{DOH}^{(\mathrm{c})}$ \\
\hline & Liver $^{(d)}$ & 5 & $\mathrm{BE}(2012)$ & Oct-Nov & ICP-MS, Hg-CVAA \\
\hline \multicolumn{6}{|l|}{ Background - Priest } \\
\hline \multirow[t]{4}{*}{ Rapids/Wanapum Pools } & Whitefish & & & & \\
\hline & Fillet & 5 & BE (2011) & November & Gamma Scan \\
\hline & Carcass & 5 & BE (2011) & November & ${ }^{90} \mathrm{Sr}$ \\
\hline & Liver $^{(\mathrm{d})}$ & 5 & BE (2011) & November & ICP-MS, Hg-CVAA \\
\hline \multirow[t]{8}{*}{$100-\mathrm{N}$ to $100-\mathrm{D}$} & Whitefish & & & & \\
\hline & Fillet & 5 & BE (2011) & Oct-Nov & Gamma Scan/DOH ${ }^{(\mathrm{c})}$ \\
\hline & Carcass & 5 & BE (2011) & Oct-Nov & ${ }^{90} \mathrm{Sr} / \mathrm{DOH}^{(\mathrm{c})}$ \\
\hline & Liver $^{(\mathrm{d})}$ & 5 & BE (2011) & Oct-Nov & ICP-MS, Hg-CVAA \\
\hline & Carp & & & & \\
\hline & Fillet & 5 & BE (2012) & April-July & Gamma Scan/DOH ${ }^{(c)}$ \\
\hline & Carcass & 5 & BE (2012) & April-July & ${ }^{90} \mathrm{Sr} / \mathrm{DOH}^{(\mathrm{c})}$ \\
\hline & Liver $^{(\mathrm{d})}$ & 5 & BE (2012) & April-July & ICP-MS, Hg-CVAA \\
\hline
\end{tabular}

(a) Refer to Figure 4.2, "2011 Wildlife Sampling Locations" identifying those locations scheduled for collection in 2011.

(b) Samples are collected in 2011 according to their specified frequency unless otherwise noted.

(c) Additional whole fish sample provided to the DOH.

(d) Ecological assessment sample. 


\subsubsection{Geese}

\begin{tabular}{|c|c|c|c|c|c|}
\hline Location $^{(a)}$ & $\begin{array}{l}\text { Species/ } \\
\text { Sample }\end{array}$ & $\begin{array}{l}\text { Number } \\
\text { of Samples }\end{array}$ & Frequency & $\begin{array}{l}\text { Collection } \\
\text { Period }\end{array}$ & Analyses \\
\hline \multirow[t]{4}{*}{100 Areas } & Canada Goose & & & & \\
\hline & Muscle & 5 & $\mathrm{BE}(2011)$ & May-July & Gamma Scan \\
\hline & Bone & 5 & $\mathrm{BE}(2011)$ & May-July & ${ }^{90} \mathrm{Sr}$ \\
\hline & Liver $^{(b)}$ & 5 & BE (2011) & May-July & ICP-MS, Hg-CVAA \\
\hline \multirow[t]{4}{*}{$\begin{array}{l}\text { Hanf Townsite to } \\
300 \text { Area }\end{array}$} & Canada Goose & & & & \\
\hline & Muscle & 5 & BE (2011) & May-July & Gamma Scan \\
\hline & Bone & 5 & $\mathrm{BE}(2011)$ & May-July & ${ }^{90} \mathrm{Sr}$ \\
\hline & Liver $^{(b)}$ & 5 & BE (2011) & May-July & ICP-MS, Hg-CVAA \\
\hline \multirow{5}{*}{$\begin{array}{l}\text { Background - } \\
\text { Desert Aire/Vantage }\end{array}$} & & & & & \\
\hline & Canada Goose & & & & \\
\hline & Muscle & 5 & BE (2011) & May-July & Gamma Scan \\
\hline & Bone & 5 & $\mathrm{BE}(2011)$ & May-July & ${ }^{90} \mathrm{Sr}$ \\
\hline & Liver $^{(b)}$ & 5 & $\mathrm{BE}(2011)$ & May-July & ICP-MS, Hg-CVAA \\
\hline $\begin{array}{l}\text { (a) Refer to Figure } \\
\text { (b) Ecological asses }\end{array}$ & $\begin{array}{l}2011 \text { Wildlife } \\
\text { t sample. }\end{array}$ & ing Locat & & & \\
\hline
\end{tabular}

\subsubsection{Upland Game Birds}

\begin{tabular}{|c|c|c|c|c|c|}
\hline Location & $\begin{array}{l}\text { Species/ } \\
\text { Sample }^{(a)}\end{array}$ & $\begin{array}{c}\text { Number } \\
\text { of Samples }\end{array}$ & Frequency & $\begin{array}{l}\text { Collection } \\
\text { Period }\end{array}$ & Analyses/Agency \\
\hline \multirow[t]{4}{*}{$100-\mathrm{D}$ to $100-\mathrm{H}$} & Pheasant & & & & \\
\hline & Muscle & 4 & BE (2012) & September & Gamma Scan \\
\hline & Bone & 4 & BE (2012) & September & ${ }^{90} \mathrm{Sr}$ \\
\hline & Liver $^{(b)}$ & 4 & BE (2012) & September & ICP-MS \\
\hline \multirow[t]{4}{*}{$100-\mathrm{H}$ to $100-\mathrm{F}$} & Pheasant & & & & \\
\hline & Muscle & 6 & BE (2012) & September & Gamma Scan/DOH ${ }^{(\mathrm{c})}$ \\
\hline & Bone & 6 & BE (2012) & September & ${ }^{90} \mathrm{Sr} / \mathrm{DOH}^{(\mathrm{c})}$ \\
\hline & Liver $^{(b)}$ & 6 & BE (2012) & September & ICP-MS \\
\hline \multirow[t]{4}{*}{ Background } & Pheasant & & & & \\
\hline & Muscle & 5 & BE (2012) & September & Gamma Scan/DOH ${ }^{(\mathrm{c})}$ \\
\hline & Bone & 5 & BE (2012) & September & ${ }^{90} \mathrm{Sr} / \mathrm{DOH}^{(\mathrm{c})}$ \\
\hline & Liver $^{(\mathrm{b})}$ & 5 & BE (2012) & September & ICP-MS \\
\hline $\begin{array}{l}\text { Pheasants pr } \\
\text { Ecological a } \\
\text { Additional }\end{array}$ & $\begin{array}{l}\text { chukars or q } \\
\text { nt sample. } \\
\text { d sample pro }\end{array}$ & $\begin{array}{l}\text { ptable if phe } \\
\text { the DOH. }\end{array}$ & ts are unava & & \\
\hline
\end{tabular}




\subsubsection{Rabbits}

\begin{tabular}{|c|c|c|c|c|c|}
\hline Location $^{\text {(a) }}$ & $\begin{array}{l}\text { Species/ } \\
\text { Sample }\end{array}$ & $\begin{array}{c}\text { Number } \\
\text { of Samples }\end{array}$ & Frequency & $\begin{array}{l}\text { Collection } \\
\text { Period }\end{array}$ & Analyses/Agency \\
\hline \multirow{4}{*}{$100 \mathrm{~N}$ Area } & Cottontail & & & & \\
\hline & Muscle & 4 & BE (2011) & Jan-Dec & Gamma Scan/DOH ${ }^{(b)}$ \\
\hline & Bone & 4 & BE (2011) & Jan-Dec & ${ }^{90} \mathrm{Sr} / \mathrm{DOH}^{(\mathrm{b})}$ \\
\hline & Liver $^{(c)}$ & 4 & BE (2011) & Jan-Dec & ICP-MS, Hg-CVAA \\
\hline \multirow[t]{4}{*}{200 E Area } & Cottontail & & & & \\
\hline & Muscle & 4 & BE (2011) & Jan-Dec & Gamma Scan/DOH ${ }^{(b)}$ \\
\hline & Bone & 4 & BE (2011) & Jan-Dec & ${ }^{90} \mathrm{Sr} / \mathrm{DOH}^{(\mathrm{b})}$ \\
\hline & Liver $^{(\mathrm{c})}$ & 4 & BE (2011) & Jan-Dec & $\mathrm{Pu}, \mathrm{ICP}-\mathrm{MS}$ \\
\hline \multirow[t]{4}{*}{200 West } & Cottontail & & & & \\
\hline & Muscle & 4 & BE (2011) & Jan-Dec & Gamma Scan/DOH ${ }^{(b)}$ \\
\hline & Bone & 4 & BE (2011) & Jan-Dec & ${ }^{90} \mathrm{Sr} / \mathrm{DOH}^{(\mathrm{b})}$ \\
\hline & Liver $^{(c)}$ & 4 & BE (2011) & Jan-Dec & $\mathrm{Pu}, \mathrm{ICP}-\mathrm{MS}$ \\
\hline \multirow[t]{4}{*}{ Background } & Cottontail & & & & \\
\hline & Muscle & 5 & BE (2011) & Jan-Dec & Gamma Scan \\
\hline & Bone & 5 & BE (2011) & Jan-Dec & ${ }^{90} \mathrm{Sr}$ \\
\hline & Liver $^{(\mathrm{c})}$ & 5 & BE (2011) & Jan-Dec & $\mathrm{Pu}, \mathrm{ICP}-\mathrm{MS}, \mathrm{Hg}-\mathrm{CVAA}$ \\
\hline
\end{tabular}

(a) Refer to Figure 4.2, "2011 Wildlife Sampling Locations."

(b) Additional whole rabbit sample provided to the $\mathrm{DOH}$.

(c) Ecological assessment sample.

\subsubsection{Deer/Elk}

\begin{tabular}{|c|c|c|c|c|c|}
\hline Location & $\begin{array}{l}\text { Species/ } \\
\text { Sample }\end{array}$ & $\begin{array}{c}\text { Number } \\
\text { of Samples }\end{array}$ & Frequency & $\begin{array}{c}\text { Collection } \\
\text { Period } \\
\end{array}$ & Analyses/Agency \\
\hline \multirow[t]{4}{*}{$100 \mathrm{~N}$ Area } & Mule Deer & & & & \\
\hline & Muscle & 2 & BE (2010) & Nov-Dec & Gamma Scan/DOH ${ }^{(a)}$ \\
\hline & Bone & 2 & BE (2010) & Nov-Dec & ${ }^{90} \mathrm{Sr} / \mathrm{DOH}^{(\mathrm{a})}$ \\
\hline & Liver $^{(b)}$ & 2 & BE (2010) & Nov-Dec & ICP-MS \\
\hline \multirow[t]{4}{*}{200 Areas } & Mule Deer & & & & \\
\hline & Muscle & 2 & BE (2010) & Nov-Dec & Gamma Scan/DOH ${ }^{(a)}$ \\
\hline & Bone & 2 & BE (2010) & Nov-Dec & ${ }^{90} \mathrm{Sr} / \mathrm{DOH}^{(\mathrm{a})}$ \\
\hline & Liver $^{(\mathrm{b})}$ & 2 & BE (2010) & Nov-Dec & $\mathrm{Pu}, \mathrm{ICP}-\mathrm{MS}$ \\
\hline \multicolumn{6}{|c|}{ Road Kill at Onsite } \\
\hline \multirow[t]{3}{*}{ Locations $^{(\mathrm{c})}$} & Mule Deer or Elk & & & & \\
\hline & Muscle & 10 & BE (2010) & As Available & Gamma Scan \\
\hline & Bone & 10 & BE (2010) & As Available & ${ }^{90} \mathrm{Sr}$ \\
\hline \multirow[t]{4}{*}{ Background $^{(d)}$} & Mule Deer & & & & \\
\hline & Muscle & 2 & BE (2010) & October & Gamma Scan/DOH ${ }^{(a)}$ \\
\hline & Bone & 2 & BE (2010) & October & ${ }^{90} \mathrm{Sr} / \mathrm{DOH}^{(\mathrm{a})}$ \\
\hline & Liver $^{(b)}$ & 2 & $\mathrm{BE}(2010)$ & October & $\mathrm{Pu}, \mathrm{ICP}-\mathrm{MS}$ \\
\hline $\begin{array}{l}\text { (a) Additional s } \\
\text { (b) Ecological a } \\
\text { (c) As available } \\
\text { (d) One of the ty }\end{array}$ & $\begin{array}{l}\text { rovided to the DOH } \\
\text { nt sample. } \\
\text { ing to location. } \\
\text { ground samples ob }\end{array}$ & ed from the & $\mathrm{OH}$. & & \\
\hline
\end{tabular}




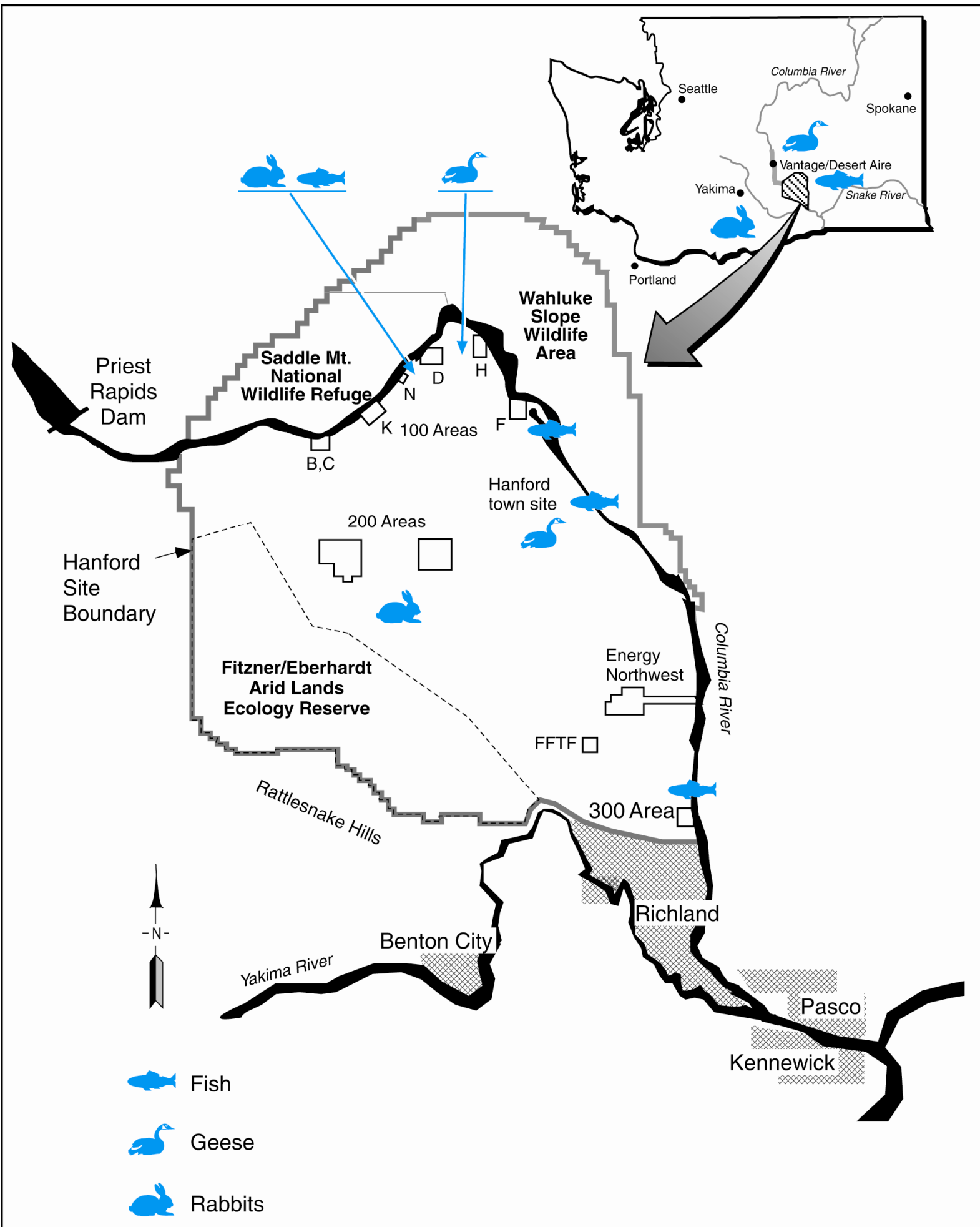

Not to Scale

2010-12-0010-3A

Figure 4.2. 2011 Wildlife Sampling Locations 


\subsection{Soil and Vegetation}

\subsection{Soil}

\begin{tabular}{|c|c|c|c|}
\hline Location & Frequency $^{(a)}$ & Collection Period & Analyses/Agency \\
\hline $100 \mathrm{~K}$ Area & 3 to $5 \mathrm{yrs}$ & June-Sept & Gamma Scan, ${ }^{90} \mathrm{Sr}, \mathrm{U}, \mathrm{Pu} / \mathrm{DOH}^{(\mathrm{b})}$ \\
\hline NE of $100 \mathrm{~N}$ Area & 3 to $5 \mathrm{yrs}$ & June-Sept & Gamma Scan, ${ }^{90} \mathrm{Sr}, \mathrm{U}, \mathrm{Pu}$ \\
\hline E of $100 \mathrm{~N}$ Area & 3 to $5 \mathrm{yrs}$ & June-Sept & Gamma Scan, ${ }^{90} \mathrm{Sr}, \mathrm{U}, \mathrm{Pu} / \mathrm{DOH}^{(\mathrm{b})}$ \\
\hline 100N Shore Above HGP & 3 to 5 yrs & June-Sept & Gamma Scan, ${ }^{90} \mathrm{Sr}, \mathrm{U}, \mathrm{Pu}$ \\
\hline 100N Spring Shoreline & 3 to $5 \mathrm{yrs}$ & June-Sept & Gamma Scan, ${ }^{90} \mathrm{Sr}, \mathrm{U}, \mathrm{Pu}$ \\
\hline Above 100D Pumphouse & 3 to $5 \mathrm{yrs}$ & June-Sept & Gamma Scan, ${ }^{90} \mathrm{Sr}, \mathrm{U}, \mathrm{Pu}$ \\
\hline 100 Area Fire Stat & 3 to $5 \mathrm{yrs}$ & June-Sept & Gamma Scan, ${ }^{90} \mathrm{Sr}, \mathrm{U}, \mathrm{Pu}$ \\
\hline $200 \mathrm{ENC}$ & 3 to 5 yrs & June-Sept & Gamma Scan, ${ }^{90} \mathrm{Sr}, \mathrm{U}, \mathrm{Pu}$ \\
\hline $\mathrm{E}$ of $200 \mathrm{E}$ & 3 to 5 yrs & June-Sept & Gamma Scan, ${ }^{90} \mathrm{Sr}, \mathrm{U}, \mathrm{Pu} / \mathrm{DOH}^{(\mathrm{b})}$ \\
\hline 200 ESE & 3 to $5 \mathrm{yrs}$ & June-Sept & Gamma Scan, ${ }^{90} \mathrm{Sr}, \mathrm{U}, \mathrm{Pu},{ }^{241} \mathrm{Am}$ \\
\hline S of $200 \mathrm{E}$ & 3 to $5 \mathrm{yrs}$ & June-Sept & Gamma Scan, ${ }^{90} \mathrm{Sr}, \mathrm{U}, \mathrm{Pu}$ \\
\hline $\mathrm{SW}$ of $\mathrm{B} / \mathrm{C}$ Cribs & 3 to 5 yrs & June-Sept & Gamma Scan, ${ }^{90} \mathrm{Sr}, \mathrm{U}, \mathrm{Pu},{ }^{241} \mathrm{Am} / \mathrm{DOH}^{(\mathrm{b})}$ \\
\hline E of $200 \mathrm{~W}$ Gate & 3 to $5 \mathrm{yrs}$ & June-Sept & Gamma Scan, ${ }^{90} \mathrm{Sr}, \mathrm{U}, \mathrm{Pu},{ }^{241} \mathrm{Am} / \mathrm{DOH}^{(\mathrm{b})}$ \\
\hline S of $200 \mathrm{~W}$ & 3 to $5 \mathrm{yrs}$ & June-Sept & Gamma Scan, ${ }^{90} \mathrm{Sr}, \mathrm{U}, \mathrm{Pu} / \mathrm{DOH}^{(\mathrm{b})}$ \\
\hline Rattlesnake Springs & 3 to $5 \mathrm{yrs}$ & June-Sept & Gamma Scan, ${ }^{90} \mathrm{Sr}, \mathrm{U}, \mathrm{Pu} / \mathrm{DOH}^{(\mathrm{b})}$ \\
\hline Yakima Barricade & 3 to $5 \mathrm{yrs}$ & June-Sept & Gamma Scan, ${ }^{90} \mathrm{Sr}, \mathrm{U}, \mathrm{Pu}$ \\
\hline $400 \mathrm{E}$ & 3 to 5 yrs & June-Sept & Gamma Scan, ${ }^{90} \mathrm{Sr}, \mathrm{U}, \mathrm{Pu}$ \\
\hline SE Side of FFTF & 3 to 5 yrs & June-Sept & Gamma Scan, ${ }^{90} \mathrm{Sr}, \mathrm{U}, \mathrm{Pu} / \mathrm{DOH}^{(\mathrm{b})}$ \\
\hline North of 300 Area & 3 to $5 \mathrm{yrs}$ & June-Sept & Gamma Scan, ${ }^{90} \mathrm{Sr}, \mathrm{U}, \mathrm{Pu} / \mathrm{DOH}^{(\mathrm{b})}$ \\
\hline South of 300 Area & 3 to $5 \mathrm{yrs}$ & June-Sept & Gamma Scan, ${ }^{90} \mathrm{Sr}, \mathrm{U}, \mathrm{Pu} / \mathrm{DOH}^{(\mathrm{b})}$ \\
\hline Hanford Townsite & 3 to 5 yrs & June-Sept & Gamma Scan, ${ }^{90} \mathrm{Sr}, \mathrm{U}, \mathrm{Pu}$ \\
\hline Wye Barricade ${ }^{(c)}$ & 3 to $5 \mathrm{yrs}$ & June-Sept & Gamma Scan, ${ }^{90} \mathrm{Sr}, \mathrm{U}, \mathrm{Pu}$ \\
\hline Prosser Barricade & 3 to $5 \mathrm{yrs}$ & June-Sept & Gamma Scan, ${ }^{90} \mathrm{Sr}, \mathrm{U}, \mathrm{Pu}$ \\
\hline ALE Field Lab ${ }^{(\mathrm{d})}$ & 3 to $5 \mathrm{yrs}$ & June-Sept & Gamma Scan, ${ }^{90} \mathrm{Sr}, \mathrm{U}, \mathrm{Pu}$ \\
\hline N End Vernita Bridge & 3 to $5 \mathrm{yrs}$ & June-Sept & Gamma Scan, ${ }^{90} \mathrm{Sr}, \mathrm{U}, \mathrm{Pu}$ \\
\hline Wahluke Slope & 3 to 5 yrs & June-Sept & Gamma Scan, ${ }^{90} \mathrm{Sr}, \mathrm{U}, \mathrm{Pu}$ \\
\hline Berg Ranch & 3 to 5 yrs & June-Sept & Gamma Scan, ${ }^{90} \mathrm{Sr}, \mathrm{U}, \mathrm{Pu}$ \\
\hline Ringold Area & 3 to $5 \mathrm{yrs}$ & June-Sept & Gamma Scan, ${ }^{90} \mathrm{Sr}, \mathrm{U}, \mathrm{Pu}$ \\
\hline W End of Fir Road & 3 to $5 \mathrm{yrs}$ & June-Sept & Gamma Scan, ${ }^{90} \mathrm{Sr}, \mathrm{U}, \mathrm{Pu}$ \\
\hline Taylor Flats No. 2 & 3 to $5 \mathrm{yrs}$ & June-Sept & Gamma Scan, ${ }^{90} \mathrm{Sr}, \mathrm{U}, \mathrm{Pu}$ \\
\hline Sagemoor Farm ${ }^{(c)}$ & 3 to 5 yrs & June-Sept & Gamma Scan, ${ }^{90} \mathrm{Sr}, \mathrm{U}, \mathrm{Pu},{ }^{241} \mathrm{Am}$ \\
\hline Byers Landing & 3 to 5 yrs & June-Sept & Gamma Scan, ${ }^{90} \mathrm{Sr}, \mathrm{U}, \mathrm{Pu}$ \\
\hline Riverview-Harris & 3 to $5 \mathrm{yrs}$ & June-Sept & Gamma Scan, ${ }^{90} \mathrm{Sr}, \mathrm{U}, \mathrm{Pu}$ \\
\hline Benton City & 3 to $5 \mathrm{yrs}$ & June-Sept & Gamma Scan, ${ }^{90} \mathrm{Sr}, \mathrm{U}, \mathrm{Pu}$ \\
\hline Sunnyside & 3 to 5 yrs & June-Sept & Gamma Scan, ${ }^{90} \mathrm{Sr}, \mathrm{U}, \mathrm{Pu},{ }^{241} \mathrm{Am}$ \\
\hline McNary Dam & 3 to 5 yrs & June-Sept & Gamma Scan, ${ }^{90} \mathrm{Sr}, \mathrm{U}, \mathrm{Pu}$ \\
\hline Walla Walla & 3 to $5 \mathrm{yrs}$ & June-Sept & Gamma Scan, ${ }^{90} \mathrm{Sr}, \mathrm{U}, \mathrm{Pu}$ \\
\hline Washtucna & 3 to $5 \mathrm{yrs}$ & June-Sept & Gamma Scan, ${ }^{90} \mathrm{Sr}, \mathrm{U}, \mathrm{Pu}$ \\
\hline Toppenish & 3 to $5 \mathrm{yrs}$ & June-Sept & Gamma Scan, ${ }^{90} \mathrm{Sr}, \mathrm{U}, \mathrm{Pu}$ \\
\hline George & 3 to 5 yrs & June-Sept & Gamma Scan, ${ }^{90} \mathrm{Sr}, \mathrm{U}, \mathrm{Pu} / \mathrm{DOH}^{(\mathrm{b})}$ \\
\hline Othello & 3 to $5 \mathrm{yrs}$ & June-Sept & Gamma Scan, ${ }^{90} \mathrm{Sr}, \mathrm{U}, \mathrm{Pu} / \mathrm{DOH}^{(\mathrm{b})}$ \\
\hline Wanapum & 3 to $5 \mathrm{yrs}$ & June-Sept & Gamma Scan, ${ }^{90} \mathrm{Sr}, \mathrm{U}, \mathrm{Pu} / \mathrm{DOH}^{(\mathrm{b})}$ \\
\hline \multicolumn{4}{|c|}{$\begin{array}{l}\text { (a) Samples are collected once every } 3 \text { to } 5 \text { years and } \\
\text { (b) Additional sample provided to the DOH. } \\
\text { (c) Quality assurance samples submitted for analyses. } \\
\text { (d) Fitzner/Eberhardt Arid Lands Ecology Reserve. }\end{array}$} \\
\hline
\end{tabular}




\subsection{Vegetation}

\begin{tabular}{lccl}
\hline \multicolumn{1}{c}{ Location } & Frequency ${ }^{(\mathrm{a})}$ & Collection Period & \multicolumn{1}{c}{ Analyses/Agency } \\
\hline $100 \mathrm{~K}$ Area & 3 to $5 \mathrm{yrs}$ & June-Sept & Gamma Scan, ${ }^{90} \mathrm{Sr}, \mathrm{U}, \mathrm{Pu} / \mathrm{DOH}{ }^{(\mathrm{b})}$ \\
NE of $100 \mathrm{~N}$ Area & 3 to $5 \mathrm{yrs}$ & June-Sept & Gamma Scan, ${ }^{90} \mathrm{Sr}, \mathrm{U}, \mathrm{Pu} / \mathrm{DOH}{ }^{(\mathrm{b})}$ \\
E of $100 \mathrm{~N}$ Area & 3 to $5 \mathrm{yrs}$ & June-Sept & Gamma Scan, ${ }^{90} \mathrm{Sr}, \mathrm{U}, \mathrm{Pu}$ \\
$100 \mathrm{~N}$ Spring Shoreline & 3 to $5 \mathrm{yrs}$ & June-Sept & Gamma Scan, ${ }^{90} \mathrm{Sr}, \mathrm{U}, \mathrm{Pu}$ \\
E of 200 W Gate & 3 to $5 \mathrm{yrs}$ & June-Sept & Gamma Scan, ${ }^{90} \mathrm{Sr}, \mathrm{U}, \mathrm{Pu}$ \\
300 Area Shoreline & 3 to $5 \mathrm{yrs}$ & June-Sept & Gamma Scan, ${ }^{90} \mathrm{Sr}, \mathrm{U}, \mathrm{Pu} / \mathrm{DOH}{ }^{(\mathrm{b})}$ \\
Hanford Townsite & 3 to $5 \mathrm{yrs}$ & June-Sept & Gamma Scan, ${ }^{90} \mathrm{Sr}, \mathrm{U}, \mathrm{Pu}$ \\
Hanford Twnsite HRM28 & 3 to $5 \mathrm{yrs}$ & June-Sept & Gamma Scan, ${ }^{90} \mathrm{Sr}, \mathrm{U}, \mathrm{Pu} / \mathrm{DOH}{ }^{(\mathrm{b})}$ \\
Ringold Area & 3 to $5 \mathrm{yrs}$ & June-Sept & Gamma Scan, ${ }^{90} \mathrm{Sr}, \mathrm{U}, \mathrm{Pu}$ \\
Sagemoor Farm & 3 to $5 \mathrm{yrs}$ & June-Sept & Gamma Scan, ${ }^{90} \mathrm{Sr}, \mathrm{U}, \mathrm{Pu}$ \\
Byers Landing & 3 to $5 \mathrm{yrs}$ & June-Sept & Gamma Scan, ${ }^{90} \mathrm{Sr}, \mathrm{U}, \mathrm{Pu}$ \\
Riverview-Harris & 3 to $5 \mathrm{yrs}$ & June-Sept & Gamma Scan, ${ }^{90} \mathrm{Sr}, \mathrm{U}, \mathrm{Pu}$ \\
Sunnyside & 3 to $5 \mathrm{yrs}$ & June-Sept & Gamma Scan, ${ }^{90} \mathrm{Sr}, \mathrm{U}, \mathrm{Pu}$ \\
Toppenish & 3 to $5 \mathrm{yrs}$ & June-Sept & Gamma $\mathrm{Scan},{ }^{90} \mathrm{Sr}, \mathrm{U}, \mathrm{Pu}$ \\
George & 3 to $5 \mathrm{yrs}$ & June-Sept & Gamma Scan, ${ }^{90} \mathrm{Sr}, \mathrm{U}, \mathrm{Pu} / \mathrm{DOH}{ }^{(\mathrm{b})}$ \\
Othello & 3 to $5 \mathrm{yrs}$ & June-Sept & Gamma Scan, ${ }^{90} \mathrm{Sr}, \mathrm{U}, \mathrm{Pu} / \mathrm{DOH}{ }^{(b)}$ \\
Wanapum & 3 to $5 \mathrm{yrs}$ & June-Sept & Gamma Scan, ${ }^{90} \mathrm{Sr}, \mathrm{U}, \mathrm{Pu} / \mathrm{DOH}{ }^{(b)}$ \\
\hline
\end{tabular}

(a) Samples are collected once every 3 to 5 years and were collected in 2008. Next collection will occur in 2013.

(b) Additional sample provided to the $\mathrm{DOH}$. 


\subsection{Sediment}

\subsection{Columbia River}

\begin{tabular}{|c|c|c|}
\hline Location $^{(\mathrm{a})}$ & Frequency & Analyses/Agency \\
\hline \multicolumn{3}{|l|}{ McNary Dam } \\
\hline McNary-OR. Side Near Dam & $\mathrm{A}$ & Gamma Scan, ${ }^{90} \mathrm{Sr}, \mathrm{U}, \mathrm{Pu}, \mathrm{ICP}-\mathrm{MS}, \mathrm{Hg}-\mathrm{CVAA}, \mathrm{TOC} / \mathrm{DOH}^{(\mathrm{b})}$ \\
\hline McNary-Wash. Side Near Dam & A & Gamma Scan, ${ }^{90} \mathrm{Sr}, \mathrm{U}, \mathrm{Pu}, \mathrm{ICP}-\mathrm{MS}, \mathrm{Hg}-\mathrm{CVAA}, \mathrm{TOC} / \mathrm{DOH}^{(\mathrm{b})}$ \\
\hline \multicolumn{3}{|l|}{ Priest Rapids Dam (PRD) } \\
\hline PRD-Grant Side Near Dam & A & Gamma Scan, ${ }^{90} \mathrm{Sr}, \mathrm{U}, \mathrm{Pu}, \mathrm{ICP}-\mathrm{MS}, \mathrm{Hg}-\mathrm{CVAA}, \mathrm{TOC} / \mathrm{DOH}^{(\mathrm{b})}$ \\
\hline PRD-Yakima Side Near Dam & A & Gamma Scan, ${ }^{90} \mathrm{Sr}, \mathrm{U}, \mathrm{Pu}, \mathrm{ICP}-\mathrm{MS}, \mathrm{Hg}-\mathrm{CVAA}, \mathrm{TOC} / \mathrm{DOH}^{(\mathrm{b})}$ \\
\hline White Bluffs Slough & A & Gamma Scan, ${ }^{90} \mathrm{Sr}, \mathrm{U}, \mathrm{Pu}, \mathrm{ICP}-\mathrm{MS}, \mathrm{Hg}-\mathrm{CVAA}, \mathrm{TOC} / \mathrm{DOH}^{(\mathrm{b})}$ \\
\hline 100 F Slough & A & Gamma Scan, ${ }^{90} \mathrm{Sr}, \mathrm{U}, \mathrm{Pu}, \mathrm{ICP}-\mathrm{MS}, \mathrm{Hg}-\mathrm{CVAA}, \mathrm{TOC}$ \\
\hline Hanford Slough & A & Gamma Scan, ${ }^{90} \mathrm{Sr}, \mathrm{U}, \mathrm{Pu}, \mathrm{ICP}-\mathrm{MS}, \mathrm{Hg}-\mathrm{CVAA}$, TOC \\
\hline Richland & $\mathrm{A}$ & Gamma Scan, ${ }^{90} \mathrm{Sr}, \mathrm{U}, \mathrm{Pu}, \mathrm{ICP}-\mathrm{MS}, \mathrm{Hg}$-CVAA, TOC \\
\hline $\begin{array}{l}\text { (a) Refer to Figure } 6.1 \text {, "2011 Sed } \\
\text { (b) Additional sample provided to }\end{array}$ & $\begin{array}{l}\text { ampling Lo } \\
\text { H. }\end{array}$ & \\
\hline
\end{tabular}

\subsection{River Shoreline Springs}

\begin{tabular}{|c|c|c|c|}
\hline Location $^{(a)}$ & $\mathrm{HRM}^{(\mathrm{b})}$ & Frequency & Analyses/Agency \\
\hline 100-B Spring 38-3 & 3.8 & $\mathrm{~A}$ & Gamma Scan, ${ }^{90} \mathrm{Sr}, \mathrm{U}, \mathrm{ICP}-\mathrm{MS}, \mathrm{Hg}-\mathrm{CVAA}$ \\
\hline 100-K Spring 63-1 & 6.3 & A & Gamma Scan, ${ }^{90} \mathrm{Sr}, \mathrm{U}, \mathrm{ICP}-\mathrm{MS}, \mathrm{Hg}-\mathrm{CVAA} / \mathrm{DOH}^{(\mathrm{c})}$ \\
\hline 100-H Spring 145-1 & 14.4 & A & Gamma Scan, ${ }^{90} \mathrm{Sr}, \mathrm{U}, \mathrm{ICP}-\mathrm{MS}, \mathrm{Hg}-\mathrm{CVAA}$ \\
\hline 100-F Spring 207-1 & 21.3 & A & Gamma Scan, ${ }^{90} \mathrm{Sr}, \mathrm{U}, \mathrm{ICP}-\mathrm{MS}, \mathrm{Hg}-\mathrm{CVAA}$ \\
\hline Hanford Spr UR 28-2 ${ }^{\text {(d) }}$ & 27.8 & A & Gamma Scan, ${ }^{90} \mathrm{Sr}, \mathrm{U}, \mathrm{ICP}-\mathrm{MS}, \mathrm{Hg}-\mathrm{CVAA} / \mathrm{DOH}^{(\mathrm{c})}$ \\
\hline Hanford Spr DR 28-2 ${ }^{(\mathrm{e})}$ & 28.3 & A & Gamma Scan, ${ }^{90} \mathrm{Sr}, \mathrm{U}, \mathrm{ICP}-\mathrm{MS}, \mathrm{Hg}-\mathrm{CVAA} / \mathrm{DOH}^{(\mathrm{c})}$ \\
\hline 300 Area Spring 41-9 & 41.9 & A & Gamma Scan, U \\
\hline 300 Area Spring 42-2 & 42.1 & A & Gamma Scan, ${ }^{90} \mathrm{Sr}, \mathrm{U}, \mathrm{ICP}-\mathrm{MS}, \mathrm{Hg}-\mathrm{CVAA} / \mathrm{DOH}^{(\mathrm{c})}$ \\
\hline 300 Area Spr DR 42-2 ${ }^{(\mathrm{e})}$ & 42.4 & A & Gamma Scan, ${ }^{90} \mathrm{Sr}, \mathrm{U}, \mathrm{ICP}-\mathrm{MS}, \mathrm{Hg}-\mathrm{CVAA} / \mathrm{DOH}^{(\mathrm{c})}$ \\
\hline 300 Area Spring 42-7 & 42.7 & A & Gamma Scan, U \\
\hline Richland Spr(SRL 437-1) & 43.7 & A & Gamma Scan, U \\
\hline
\end{tabular}

(a) Refer to Figure 6.1, "2011 Sediment Sampling Locations."

(b) HRMs are a series of signposts along the Hanford Site shoreline of the Columbia River that are roughly $1.6 \mathrm{~km}$ (1 mi) apart. The Vernita Bridge is HRM \#0 and Ferry Street in Richland is HRM \#46. Samples collected between HRMs are assigned a decimal.

(c) Additional sample provided to the $\mathrm{DOH}$.

(d) $\mathrm{UR}=$ Upriver from noted location.

(e) $\mathrm{DR}=$ Downriver from noted location.

\subsection{Onsite Pond}

\begin{tabular}{lrc}
\hline \multicolumn{2}{c}{ Location $^{\text {(a) }}$} & Analyses/Agency \\
\hline West Lake & SA (Feb \& June) $\quad$ Gamma Scan, ${ }^{90} \mathrm{Sr}, \mathrm{U},{ }^{99} \mathrm{Tc}$, Alpha, Beta \\
\hline (a) Refer to Figure 6.1, "2011 Sediment Sampling Locations.” \\
\hline
\end{tabular}




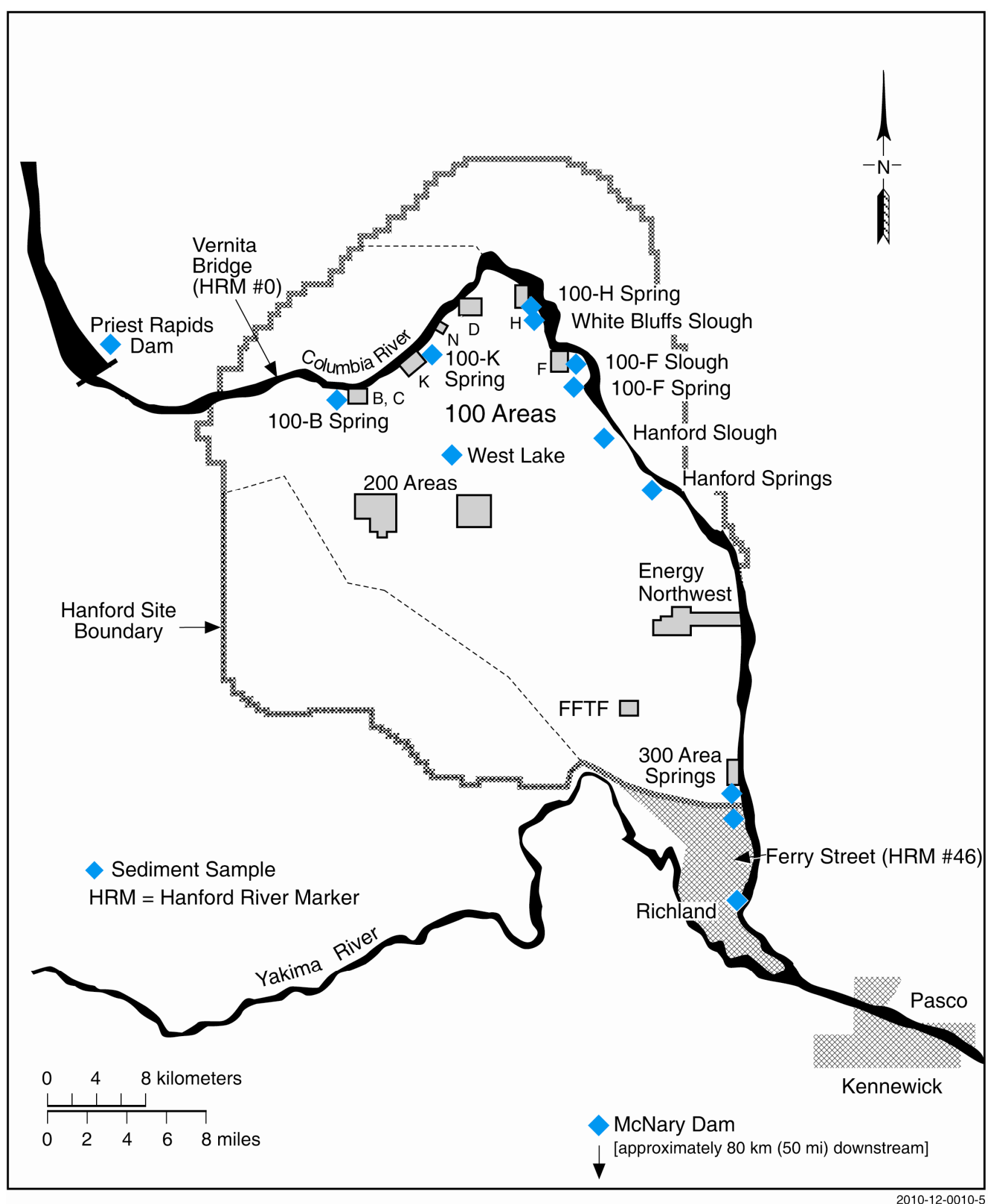

Figure 6.1. 2011 Sediment Sampling Locations 
PNNL-20121

\section{Distribution}

No. of

Copies

\section{OFFSITE (34)}

2 L. Albin

Division of Radiation Protection

Washington State Department of Health

P.O. Box 47827

Olympia, WA 98504-7827

L. Albright

U.S. Fish and Wildlife Service

64 Maple Street

Burbank, WA 99323

C. Andrade

National Oceanic and Atmospheric

Administration

DARC 7600

Sandpoint Way NE

Seattle, WA 98115

J. Bartoszek

U.S. Fish and Wildlife Service

64 Maple Street

Burbank, WA 99323

J. Bell

Nez Perce Tribe

P.O. Box 365

Lapwai, ID 83540

G. Bohnee

Nez Perce Tribe

P.O. Box 365

Lapwai, ID 83540

R. Brunoe, GW Natural Resource

Confederated Tribes of Warm Springs

Reservation

P.O. Box 1299

Warm Springs, OR 97761

L. Buck

Wanapum

P.O. Box 878

Ephrata, WA 98823
No. of

Copies

R. Buck, Jr.

Wanapum

P.O. Box 878

Ephrata, WA 98823

L. Buelow

U.S. Environmental Protection Agency, 309 Bradley, Suite 115, MS B1-46

Richland, WA 99352

J. Carleton

Washington Department of Fish and Wildlife

600 Capitol Way

1111Washington Street SE

Olympia, WA 98501-1091

D. Engstrom

Oregon Department of Energy

625 Marion Street NE

Salem, OR 97301-3737

L. Gadbois

U.S. Environmental Protection Agency

309 Bradley, Suite 115, MS B1-46

Richland, WA 99352

L. Goldstein

Washington State Department of Ecology

P.O. Box 47600

Olympia, WA 98504

B. Harper

Confederated Tribes of the Umatilla Indian

Reservation

750 Swift, Suite 14

Richland, WA 99352

S. Harris

Confederated Tribes of the Umatilla Indian

Reservation

P.O. Box 638

Pendleton, OR 97801

Distr. 1 
No. of

\section{Copies}

G. Hughes

U.S. Fish and Wildlife Service

64 Maple Street

Burbank, WA 99323

R. Jim, Manager

Yakama Nation

2808 Main Street

Union Gap, WA 98903

E. Kramer

Division of Radiation Protection

Washington State Department of Health

P.O. Box 47827

Olympia, WA 98504-7827

D. Landeen

Hanford Natural Resources Trustee

Council

Nez Perce Tribe

P.O. Box 365

Lapwai, ID 83540

J. McConnaughey

Yakama Nation

P.O. Box 6066

Kennewick, WA 99336-0066

J. McDonald

Washington State Department of Health 309 Bradley, Suite 201

Richland, WA 99352

A. Nomee

Department of Natural Resources

Coeur d'Alene Tribe

P.O. Box 408

Plummer, ID 83851-9703

Office of Environmental Cleanup

Director - Hanford Project Office

$12006^{\text {th }}$ Avenue

Seattle, WA 98101

C. Pleasants

Confederated Tribes of the Colville Indian

Reservation

P.O. Box 150

Nespelem, WA 99155
No. of

Copies

M. Priddy

Washington State Department of Health

309 Bradley, Suite 201

Richland, WA 99352

P. Rigdon

Yakama Nation

P.O. Box 151

Toppenish, WA 98948

T. Rogers

Washington State Department of Health

309 Bradley, Suite 201

Richland, WA 99352

B. Ruben

Washington State Department of Health 309 Bradley, Suite 201

Richland, WA 99352

P. Shaffer

Oregon Department of Energy

625 Marion Street NE

Salem, OR 97301-3737

J. Skriletz

Washington Department of Fish and

Wildlife

600 Capitol Way

1111 Washington Street NE

Olympia, WA 98501-1091

T. Southworth

Energy Northwest

P.O. Box 968

Richland, WA 99354

S.P. Van Verst

Division of Radiation Protection

Washington State Department of Health

P.O. Box 47827

Olympia, WA 98504-7827

\section{ONSITE (76)}

\section{CH2M HILL Plateau Remediation} Company

N.A. Homan

H8-45 
No. of

Copies

DOE Pacific Northwest Site Office

J. Erickson K9-42

22 DOE Richland Operations Office

N. Ceto

R.J. Corey

B.L. Foley

J.R. Franco

W.M. Glines

J.A. Hansen

R.G. Hastings

A.R. Hawkins

J.P. Sands

K.M. Thompson

A.C. Tortoso

G.T. Tyree

D.C. Ward (5)

J.L. Ward

S.R. Weil

J. Zeisloft

DOE Public Reading Room (2)

A7-50

A5-14

A6-38

A3-04

A5-17

A5-11

A5-19

A5-14

A3-04

A6-38

A6-38

A $7-75$

A5-15

A $7-50$

A5-15

A3-04

$\mathrm{H} 2-53$

3 DOE Office of River Protection

J.A. Dowell

H6-60

L.A. Huffman

H6-60

W. Russell

H6-60

8 Mission Support Alliance, LLC

J.J. Dorian

H7-28

L.L. Fritz

H7-26
No. of

Copies

A.R. Johnson R3-12

L.M. Kelly R3-15

S.M. McKinney H7-28

C.J. Perkins H7-28

K.A. Peterson H7-28

D.D. Teel H7-28

2 Washington Closure Hanford, LLC

L.C. Hulstrom

H4-22

R.W. Ovink

H4-21

39 Pacific Northwest National Laboratory

E.J. Antonio

K3-54

M. Barnett

J2-25

L.E. Bisping (20)

K6-75

J.M. Brandenberger

Sequim

R.L. Dirkes (2)

K6-75

J.L. Downs

K6-85

R.E. Durham

K6-85

B.G. Fritz

K6-75

K.D. Hand

K6-85

B.D. Johns

K6-75

B.K. Lasorsa

E.A. Lepel

Sequim

R.P. Mueller

P8-01

B.E. Opitz

K6-85

G.W. Patton

K6-75

R.E. Peterson

K6-75

T.M. Poston

K6-75

J.A. Stegen

K6-75

K3-66

Historical File-T.M. Poston

K6-75 


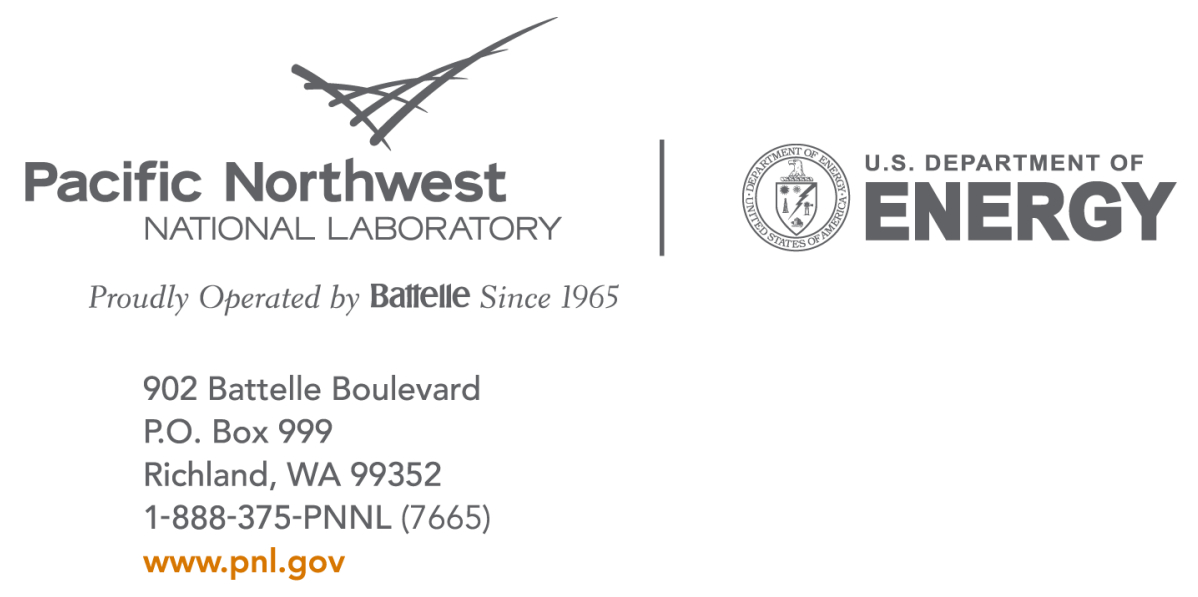

